\title{
Sinking during earthquakes: Critical acceleration criteria control drained soil liquefaction
}

\author{
C. Clément, ${ }^{1}$ R. Toussaint,,${ }^{1,2, *}$ M. Stojanova, ${ }^{1,3}$ and E. Aharonov ${ }^{4}$ \\ ${ }^{1}$ Institut de Physique du Globe de Strasbourg, Université de Strasbourg, CNRS, UMR 7516, 67084 Strasbourg Cedex, France \\ ${ }^{2}$ PoreLab, Department of Physics, University of Oslo, 0316 Oslo, Norway \\ ${ }^{3}$ Institut Lumière Matière, Université Lyon 1, CNRS, UMR 5586, 69361 Lyon Cedex 07, France \\ ${ }^{4}$ Fredy and Nadine Herrmann Institute of Earth Sciences, Hebrew University of Jerusalem, Jerusalem 91904, Israel
}

(Received 12 April 2017; published 21 February 2018)

\begin{abstract}
This article focuses on liquefaction of saturated granular soils, triggered by earthquakes. Liquefaction is defined here as the transition from a rigid state, in which the granular soil layer supports structures placed on its surface, to a fluidlike state, in which structures placed initially on the surface sink to their isostatic depth within the granular layer. We suggest a simple theoretical model for soil liquefaction and show that buoyancy caused by the presence of water inside a granular medium has a dramatic influence on the stability of an intruder resting at the surface of the medium. We confirm this hypothesis by comparison with laboratory experiments and discrete-element numerical simulations. The external excitation representing ground motion during earthquakes is simulated via horizontal sinusoidal oscillations of controlled frequency and amplitude. In the experiments, we use particles only slightly denser than water, which as predicted theoretically increases the effect of liquefaction and allows clear depth-ofsinking measurements. In the simulations, a micromechanical model simulates grains using molecular dynamics with friction between neighbors. The effect of the fluid is captured by taking into account buoyancy effects on the grains when they are immersed. We show that the motion of an intruder inside a granular medium is mainly dependent on the peak acceleration of the ground motion and establish a phase diagram for the conditions under which liquefaction happens, depending on the soil bulk density, friction properties, presence of water, and peak acceleration of the imposed large-scale soil vibrations. We establish that in liquefaction conditions, most cases relax toward an equilibrium position following an exponential in time. We also show that the equilibrium position itself, for most liquefaction regimes, corresponds to the isostatic equilibrium of the intruder inside a medium of effective density. The characteristic time to relaxation is shown to be essentially a function of the peak ground velocity.
\end{abstract}

DOI: 10.1103/PhysRevE.97.022905

\section{INTRODUCTION}

Under usual conditions, natural and artificial soils (used as geotechnical foundations or construction materials) support the weight of infrastructure placed on their surface and the stresses exerted on their surface are transmitted to the underlying grains along force chains [1]. However, contacts between grains may be weakened during shaking and/or by the addition of a liquid phase, which in general exerts an additional fluid pressure on the grains. When these contacts break or slide, the system is not stable anymore, so the granular medium loses its ability to support shear stress and flows as a liquid, which is referred to as liquefaction [2]. In such cases debris flows, avalanches, quicksand, or liquefaction can occur. Buildings on liquefied soils may sink or tilt, and pipelines are displaced or float to the surface. All of the above phenomena may lead to significant damage.

In this paper we focus on soil liquefaction associated with earthquakes [2-4]. Some areas are well known to be prone to soil liquefaction, like the New Madrid Seismic Zone in the central United States or Mexico city in Mexico [2,5]. The last major earthquakes which have been followed by severe liquefaction effects, listed in [4], are the 1964 Alaska earthquake of 9.2 moment magnitude (Mw) [6], the 1964

${ }^{*}$ Corresponding author: renaud.toussaint@ unistra.fr
Niigata earthquake of 7.5 Mw [7,8] in Japan, and the 2011 Christchurch earthquake of 6.3 Mw [9] in New Zealand.

Liquefaction was historically first explained by Terzaghi [10], relating liquefaction occurrence to the effective stress in the material. Further geotechnical work [11,12] improved the principle of Terzaghi in order to explain as many liquefaction cases as possible. The current understanding of liquefaction, which underlies the construction principles for foundations and roads, can be summed up as follows: During earthquakes, seismic waves disturb the grain-grain contacts and some weight initially carried by the sediment is then shifted to the interstitial pore water [2]. The consolidation of the saturated sediment occurs in effectively undrained conditions (due to the shorttime scale of earthquakes) and pore pressure builds up as the granular packing compacts. As a result, the effective stress carried by the sediment decreases. If the pore pressure rises more, the solid weight can be entirely borne by pore water and the sediment becomes fluidlike, i.e., it cannot sustain shear stress in a static configuration. This accepted mechanism thus assumes that the granular medium must lose its strength completely to produce liquefied behavior.

The above-described pore-pressure theory of earthquakeinduced soil liquefaction and its recent advances indeed explain many natural instances of observed liquefaction [12-14], yet it fails to explain many other field observations of earthquakeinduced liquefaction. Examples of types of field occurrences of 
liquefaction that are not explained by the pore-pressure theory [15] include far-field liquefaction triggered at low-energy density [16,17], liquefaction under fully drained conditions [18-20], repeated liquefaction [21], and liquefaction in precompacted soils [22].

In fact, elevated pore pressure is not the only path for granular material liquefaction. The phenomenon of solid-liquid transition of granular materials is known in a more general framework as fluidization. Fluidization of a granular medium occurs when an initially rigid medium loses its cohesion and starts behaving like a fluid. One of its most famous examples is quicksand, a granular medium which can support a body on its surface as long as the said body is not moving, whereas if it is moving, the body sinks into the quicksand [23,24].

A compact, dense granular medium at rest behaves like a solid. The grains experience friction due to the normal stress they apply to each other, traditionally coming from the gravitational loading. The friction enables the grains to resist external forces without flowing [25] and sustain weight by redistributing it along force chains [1]. The importance of normal stresses for the rigidity of a granular medium can be illustrated with some recent penetration experiments. Lohse et al. in [26] and Brizinski et al. in [27] used a homogeneous air injection to loosen the granular medium and unload some of the gravitational force on grains. After the injection was stopped, the granular medium could not carry the weight it was carrying before and any objects placed on its surface sank. In a more extreme situation, where the grains are loose and also have a very low density (expanded polystyrene), the penetration of the intruder is infinite, just like the penetration of a dense object in a liquid [28]. The external energy required for fluidization can be of different nature. It can come, for example, from gravity forces [29-31], shear stress [32], vibrations [24,33-36], or the flow of an interstitial fluid $[37,38]$ and produce granular flows that can exhibit fluidlike behavior such as buoyancy [34] or antibuoyancy [39] force, waves on their surface [33,40], flow instabilities [41-46], and size segregation [35,36].

Granular media start to behave as fluids when global contact sliding is initiated throughout the media. The force needed to initiate sliding depends on the strength of the grain contacts; for an easy fluidization, one needs to unload some of the normal stress, hence reducing the friction forces. For example, the shear stress necessary to make a granular layer flow decreases when the granular medium is vibrated [47], the vibrations weakening the granular contacts. The presence of an interstitial fluid can play a similar role. When the grains are immersed, the effective normal stress they are subjected to is lowered by the pore pressure of the fluid, hence reducing the friction forces between grains. If the pore pressure is high enough, the friction forces between grains can be completely suppressed and the medium cannot resist shear anymore [19]. Considerations on granular media have been used to generalize these liquefaction conditions on the heterogeneous pore-pressure distribution in disordered granular media [48]. In another study, Geromichalos et al. [49] show that the addition of water (more than $1 \%$ of the total volume) decreases the segregation effect inside granular media subjected to horizontal shaking, and attribute this to the fact that water makes the particles slide more easily on each other. It is important to note that it is not necessary to unload all of the normal stress on contacts (with the prime example being pore pressure reaching the normal stress value) in order to initiate liquefaction. Instead, causing sliding of contacts throughout the media is sufficient to produce features of liquefaction.

In this present study we will consider the effects of both water presence and vibrations on the behavior of a granular medium. The situation is very similar to the one in [50], where a dry granular medium was fluidized by vibration and the sinking of an intruder initially placed on the granular surface was observed. By shaking the granular medium to reproduce earthquakes we can observe that objects originally resting on the surface partly or entirely sink in this medium. The grain-grain contacts are disturbed by the shaking, which allows some grains to slide on each other. This effect is shown to be promoted by the presence of water, but does not require elevated pore pressure beyond the hydrostatic value. Our aim is to first highlight how liquefaction in such drained conditions can be explained by friction and sliding inside the medium and to characterize the liquefaction state according to the parameters of the shaking. Section II presents the research questions, the experimental material, and a simple theoretical model for the phenomenon. Section III presents the methods for the experiments and simulations, and the detailed characterization of the liquefaction regimes. Section IV presents the different results about the classification of deformation regimes as a function of the applied shaking and about the characteristic sinking velocity and equilibrium depth. A discussion of results and their consequences is presented in Sec. V.

\section{PHYSICS OF LIQUEFACTION}

The following section will provide an overview of the problem. We will qualitatively describe some of the experimental results in order to illustrate the different behavioral regimes that we observed. We will then explain the mechanisms behind these behaviors and the transition between them and identify the link with soil liquefaction.

\section{A. Description of the observed deformation regimes}

Our experiment is a simplified model of a building resting on a soil during the passage of a seismic wave. The soil is simplified to a granular medium made of nonexpanded polystyrene spheres of density $\rho_{s}=1050 \mathrm{~kg} \mathrm{~m}^{-3}$ [51] and mean diameter of $140 \mu \mathrm{m}$. It can be completely dry or completely saturated. The granular medium is in a test cell, a transparent poly(methyl methacrylate) (PMMA) box of dimensions $12.8 \times 12.8 \times 12.5 \mathrm{~cm}^{3}$. A hollow sphere of 40 $\mathrm{mm}$ diameter and of effective density of $1030 \pm 5 \mathrm{~kg} \mathrm{~m}^{-3}$ initially rests on the top of the layer (Fig. 1) representing an analog building. To reproduce the effect of an earthquake we shake the different media horizontally with a controlled frequency and amplitude. The frequency ranges from 0.15 to $50 \mathrm{~Hz}$ and the peak ground acceleration (PGA) from $10^{-2}$ to $100 \mathrm{~m} \mathrm{~s}^{-2}$, corresponding to conditions met during earthquakes with macroseismic intensity of II to V-VI [52].

We observed that the behavior of the system depends on the PGA applied to it. In the dry case, at small imposed accelerations the intruder and the particles follow the cell 


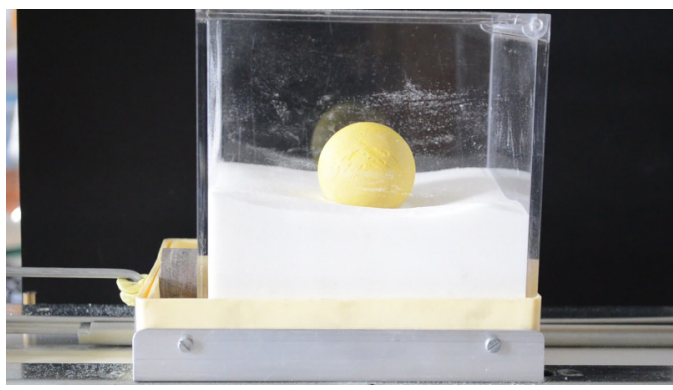

FIG. 1. Initial state at mechanical equilibrium. The intruder diameter is $40 \mathrm{~mm}$ and its density is $1030 \pm 5 \mathrm{~kg} \mathrm{~m}^{-3}$, close to the one of the grains composing the granular medium. The medium is either dry or fully saturated with water. This example shows a dry medium.

movement, but are almost immobile with respect to each other. For larger PGA, convection cells appear inside the granular medium: The particles on the top of the medium can be seen moving toward the sides. The intruder stays at the surface (see Fig. 2) and can eventually roll from side to side if the acceleration is large enough.

With an initially saturated medium, still no significant motion is observed at low shaking accelerations. However, when the acceleration is increased, the intruder sinks rapidly into the medium, until an equilibrium is reached, which can be close to a total immersion, as shown in Fig. 3. At the equilibrium the intruder remains almost immobile and the rearrangements of the particles on the surface of the medium are too small to be observed. For even larger imposed accelerations, a similar sinking of the intruder is observed, but is accompanied by motion of the surrounding grains. In this case the motion of the intruder and medium never ceases totally during the imposed oscillations.

For the saturated media, we can therefore identify three behaviors, occurring at different peak ground accelerations.

Low PGA: rigid behavior. If the acceleration of the medium is low, the system oscillates like a solid, following the cell's movement. The intruder stays at the surface and only a small descent of a few millimeters can sometimes be observed.

Intermediate PGA: heterogeneous liquefaction $(H L)$ behavior. When the acceleration is increased beyond some critical level, the intruder rapidly sinks in the saturated medium until attaining an equilibrium position where it stops moving. The

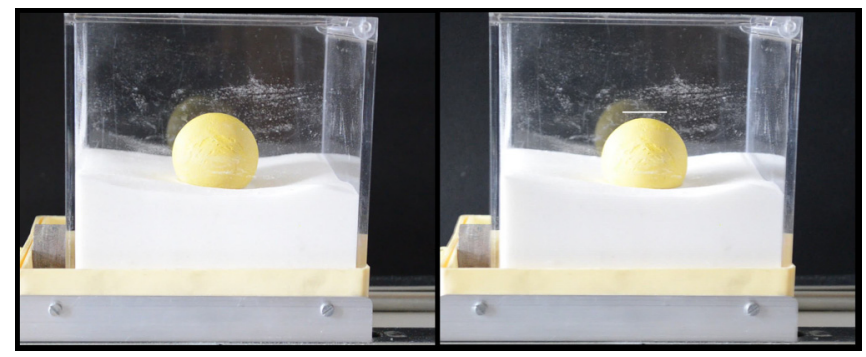

FIG. 2. Dry medium, initial state on the left and final state on the right. The intruder sinks a few percent of its diameter. The initial position is represented on the final picture by a white horizontal line. The bulk densities of the intruder and the grains composing the medium are equal.

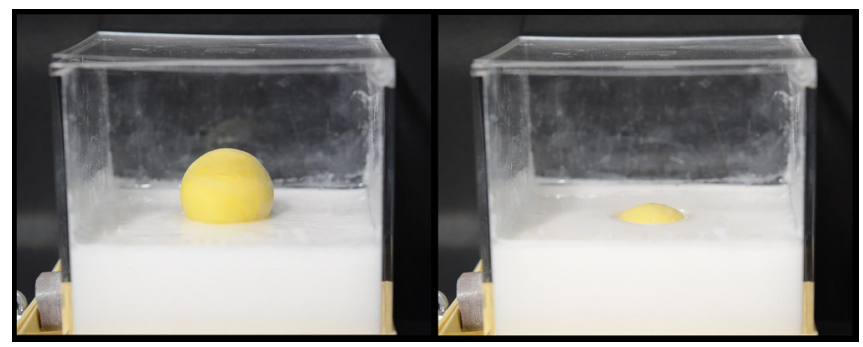

FIG. 3. Saturated medium, initial state on the left and final state on the right. The intruder is eventually almost entirely immersed. The bulk densities of the intruder and of the grains composing the medium are equal.

medium on the surface shows only little rearrangements. As will be seen later in the simulations, the grains in the intruder vicinity, underneath it, are in this case locked (not sliding on each other), the contact normal stresses rising due to the intruder weight. They accompany the motion of the intruder. In contrast, further from the intruder, the grains sometimes slide on each other, hence the term heterogeneous liquefaction, to reflect the difference between these two zones, i.e., the fact that the liquidlike behavior is heterogeneously distributed. This behavior is only observed for a saturated granular medium. In the dry case, at equal PGA the intruder stays on the surface of the granular medium.

High PGA: global excitation liquefaction (GEL) behavior. For even higher accelerations, we can observe a total and continuous rearrangement of the medium, presenting convection cells. The intruder stays at the surface of the medium for dry cases or sinks in saturated conditions. We call this behavior global excitation liquefaction because (as will be illustrated in the simulations) the whole medium is rearranged, sliding between grains can happen in the whole cell, and deformation never stops.

In our experiments, the solid behavior at low PGA corresponds to "regular" solid soil, sustaining the weight above it. The GEL behavior at high acceleration is not a phenomenon that is observed during earthquakes in nature, because it requires a very high acceleration and can only be reached during artificial excitation of granular material. In this case the fact that the intruder stays at the surface of dry granular media is related to the Brazil nut effect $[35,39,49,53,54]$. Finally, the HL behavior observed at intermediate PGA corresponds to soil liquefaction during an earthquake. In these experiments, it is the addition of water that enables the medium to liquefy. Indeed, it is only when the medium is saturated that we observe a regime where an intruder can penetrate it. The shape of the intruder also affects this behavior in dry grains, since with similar densities, cylindrical objects can sink or tilt in dry granular media [55].

\section{B. Problem definition and a simple model}

The observations described above highlight so far unreported aspects of liquefaction. In our experiments, the presence of water is crucial for observing liquefactionlike sinking of the intruder. We explain the physics of the liquefaction appearing 


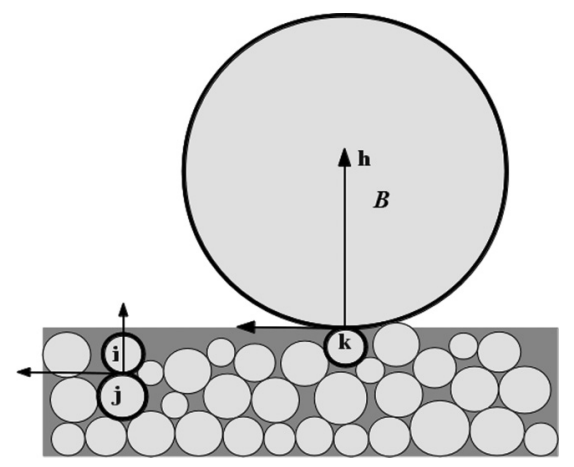

FIG. 4. Theoretical model of saturated soil with a spherical intruder on the top. The weight of particle $i$ applied on particle $j$ is reduced by the buoyancy, whereas the weight of particle $B$ (the intruder), whose center sits at height $h$, is entirely transmitted to particle $k$.

in these experiments using a simple theoretical soil consisting of a (saturated or dry) grain packing, as in Fig. 4.

This soil is composed of spherical particles and water filling the poral space between them. A large sphere on top of the granular soil represents a building built on it. We assume that the situation is initially at mechanical equilibrium. Here we will determine under which conditions this equilibrium can be broken and the large sphere could start to sink into the medium. We first focus on the saturated cases, as represented in Fig. 4.

\section{Saturated medium}

To define the sliding condition we will distinguish the case of a contact between two grains $\left(P_{i}\right.$ and $P_{j}$ on Fig. 4) or between the intruder and a grain $\left(P_{B}\right.$ and $\left.P_{k}\right)$. First consider two particles inside the saturated soil $P_{i}$ and $P_{j}$ placed on top of each other. The normal force at the contact $i j$ acting on the lower sphere $P_{j}$ is the effective weight of the column above it resulting from gravity and buoyancy force

$$
\boldsymbol{F}_{\boldsymbol{n}}^{\boldsymbol{i} \boldsymbol{j}}=M_{\mathrm{above}}\left(1-\frac{\rho_{\mathrm{w}}}{\rho_{\mathrm{s}}}\right) \boldsymbol{g},
$$

where $M_{\text {above }}$ is the mass of the grains inside the column above $P_{j}, \rho_{\mathrm{s}}$ is the particle density, $\rho_{\mathrm{w}}$ is the water density, and $\boldsymbol{g}$ is the gravitational acceleration. Next consider the intruder $P_{B}$ ( $B$ for building) and the particle of the soil right under it (or the set of grains under it and in contact with it) $P_{k}$. The normal force that $P_{B}$ applies on $P_{k}$ is

$$
\boldsymbol{F}_{\boldsymbol{n}}^{\boldsymbol{B} \boldsymbol{k}}=M_{B} \boldsymbol{g},
$$

with $M_{B}$ the mass of $P_{B}$. There is no buoyancy term in this case, since no part of the intruder is submerged under water. We apply to this soil a horizontal oscillation with a lateral displacement of the form $A \sin (\omega t)$. The peak ground acceleration due to this movement is therefore $A \omega^{2}$. We consider that the medium and intruder follow the imposed external motion, in order to check whether the contacts reach a sliding threshold, and use this as a sign of possible deformation. At low acceleration, the contact $i j$ is rigid and experiences a tangential force of the form

$$
\boldsymbol{F}_{\boldsymbol{t}}^{i j}=M_{\mathrm{above}} A \omega^{2} .
$$

We assume that each contact follows a Coulomb friction law, where $\mu$ is the internal friction coefficient equal to the tangent of the repose angle of the considered granular material. Thus, if the tangential force on the contact exceeds the criterion set by the Coulomb friction law, the contact $i j$ slides and Eq. (3) becomes $\boldsymbol{F}_{t}^{i j}=\mu \boldsymbol{F}_{n}^{i j}$. Thus the medium remains rigid if

$$
\left|M_{\text {above }} A \omega^{2}\right|<\left|\mu \boldsymbol{F}_{n}^{i j}\right|,
$$

i.e., while

$$
M_{\text {above }} A \omega^{2}<\mu M_{\text {above }}\left(1-\frac{\rho_{\mathrm{w}}}{\rho_{\mathrm{s}}}\right) g .
$$

Now, if we introduce a dimensionless peak ground acceleration, normalized by the gravitational acceleration $\Gamma=\frac{A \omega^{2}}{g}$, the preceding equation becomes

$$
\Gamma<\mu \frac{\rho_{\mathrm{s}}-\rho_{\mathrm{w}}}{\rho_{\mathrm{s}}} .
$$

While $\Gamma$ is low enough to satisfy Eq. (4), the particles inside the saturated soil do not slide on each other and the medium acts like a rigid body.

The condition for sliding of the contact between the intruder $P_{B}$ and particle under it $P_{k}$ is different: The horizontal oscillation induces a tangential force on $P_{B}$, which will slide on $P_{k}$ if and only if

$$
M_{B} A \omega^{2}>\left|\mu \boldsymbol{F}_{\boldsymbol{n}}^{\boldsymbol{B} \boldsymbol{k}}\right|=M_{B} \mu g .
$$

In other words, the emerged particle $P_{B}$ will stick on $P_{k}$ while

$$
\Gamma<\mu \text {. }
$$

If $\Gamma>\mu$ the intruder can slide on the particle below it. We can see in Eq. (5) that the acceleration $\Gamma$ required for the intruder to slide is higher than the one needed to make the immersed particles of the soil slide, Eq. (4). This is due to the presence of water which carries a non-negligible part of the particles weight through the buoyancy so that the solid pressure between them is reduced and they can slide more easily. The emerged intruder is not partially carried by water and its contact on particle $P_{k}$ is stronger. Depending on $\Gamma$ and according to the previous results, three different regimes can be defined for the granular system:

$$
\begin{gathered}
\Gamma<\mu \frac{\rho_{\mathrm{s}}-\rho_{\mathrm{w}}}{\rho_{\mathrm{s}}} \quad(\operatorname{low} \Gamma), \\
\mu \frac{\rho_{\mathrm{s}}-\rho_{\mathrm{w}}}{\rho_{\mathrm{s}}}<\Gamma<\mu \quad(\text { intermediate } \Gamma), \\
\mu<\Gamma \quad(\text { high } \Gamma) .
\end{gathered}
$$

For low accelerations, the tangential force resulting on the particles contacts is too low to make any particle slide; the medium cannot be rearranged and will behave like a solid. Hence, this regime corresponds to the solid behavior of the system observed during our experiments. For intermediate $\Gamma$, many of the small particles can slide on each other, while the intruder cannot slide on the particles beneath it. Hence, we can reasonably assume that the intruder will sink downward because the medium is being rearranged around it and that this regime corresponds to the HL case. Finally, for $\Gamma>\mu$, the intruder can also slide on the particles beneath it, hence it 
behaves like the other particles. One can reasonably suppose that it will not continuously sink in the medium, because of the Brazil nut effect $[35,39,49,53,54]$. If $\Gamma$ gets even larger, until satisfying $\Gamma>1$, the medium gets decompacted. The acceleration is then large enough to defy gravity and the particles can make short jumps (short ballistic trajectories above the connected medium). The case of $\Gamma>\mu$ corresponds to the GEL case.

Even though our model of the liquefaction system is very simple, it predicts three distinct regimes which may be identified experimentally. The rest of our work will focus on experimental and numerical verification of these predictions. We will systematically vary $\Gamma$ in order to explore the three regimes defined by this model. The case we are mostly interested in for its representativity of natural liquefaction during earthquakes is the case of intermediate $\Gamma$, where the submerged small particles can slide around a static intruder, making the intruder sink in the medium.

In the following we will refer to the theoretical boundary between the three regimes as

$$
\Gamma_{\mathrm{HL}}=\mu \frac{\rho_{\mathrm{s}}-\rho_{\mathrm{w}}}{\rho_{\mathrm{s}}}, \quad \Gamma_{\mathrm{GEL}}=\mu .
$$

\section{Dry medium}

Inside dry granular media, the buoyancy forces disappear. Since we initially considered an intruder emerged above the saturated granular medium, Eq. (5) remains correct for dry media. Equation (4), which gives the acceleration at which two particles of the soil can slide on each other, becomes

$$
\Gamma<\mu \frac{\rho_{\mathrm{s}}-0}{\rho_{\mathrm{s}}}=\mu,
$$

which is identical to Eq. (5). Hence, in the case of a dry granular medium, the sliding conditions are the same for the particles of the medium and for the intruder, provided that the friction coefficient $\mu$ is the same for grain-grain contacts and for intruder-grain contacts. The intermediate acceleration case given by Eq. (7) disappears for dry media. With an increase of $\Gamma$, this theory predicts that dry media will change their behaviors from the rigid case to the GEL case around

$$
\Gamma_{\mathrm{HL}}=\Gamma_{\mathrm{GEL}}=\mu .
$$

\section{Final intruder position in a saturated medium}

Let us consider next the final equilibrium state reached by the intruder in the saturated medium during liquefaction regimes. Assuming that vertical friction forces average to zero and only buoyancy forces and gravity dictate the final depth, the final position of the intruder can be estimated as the isostatic depth of the intruder inside a fluid of effective density $\rho_{\text {eff }}$, taking into account the particle density, the fluid density and the porosity $\Phi$. We define the effective medium density $\rho_{\text {eff }}$ as $\rho_{\text {eff }}=\Phi \rho_{\mathrm{w}}+(1-\Phi) \rho_{\mathrm{s}}$. We measure $\Phi$ in our experiments to be between 0.345 and 0.365 , which is close to a close randomly packed density [56], so that $\rho_{\text {eff }}=1032.2 \pm 0.4 \mathrm{~kg} \mathrm{~m}^{-3}$. If the final pressure profile in the granular medium is identical to a simple hydrostatic fluid situation and if the medium acts as an effective viscous fluid, the motion of the intruder is ruled by the equation

$$
V_{B} \rho_{B} g-V_{B \text { im }}(z) \rho_{\text {eff }} g-\alpha(z) \dot{z}=V_{B} \rho_{B} g \ddot{z},
$$

where $z$ is the downward pointing vertical coordinate of the center of the intruder, $V_{B \mathrm{im}}(z)$ is the immersed volume of the intruder (depending on its elevation), $\rho_{B}$ is the intruder density, and $V_{B}$ is its total volume. The first term of Eq. (12) refers to the weight of the intruder and the second term refers to the buoyancy force. Finally, $\alpha \dot{z}$ is a dissipative term due to forces exerted by the particles on the intruder, slowing down its motion. In the case of an effective medium of density $\rho_{\text {eff }}<\rho_{B}$, the intruder is supposed to sink continuously because it is denser than the effective medium, while if $\rho_{\text {eff }}>\rho_{B}$ it reaches an equilibrium set by isostasy. Since the intruder density is chosen as $1030 \mathrm{~kg} \mathrm{~m}^{-3}$ in experiments, a macroscale equilibrium state exists with these simple assumptions and the intruder is expected to sink until it is nearly entirely immersed. If this state is reached, we name $V_{B \text { im }}^{\text {equilibrium }}$ the immersed volume of the intruder under isostatic equilibrium, corresponding to

$$
V_{B} \rho_{B} g-V_{B \text { im }}^{\text {equilibrium }} \rho_{\text {eff }} g=0,
$$

giving

$$
V_{B \text { im }}^{\text {equilibrium }}=V_{B} \frac{\rho_{B}}{\rho_{\text {eff }}} .
$$

This value will be used as a theoretical reference and compared to the final immersed volume observed in our experiments and simulations.

\section{EXPERIMENTAL AND SIMULATION METHODS FOR TRACKING LIQUEFACTION}

\section{A. Presentation of the experiments}

Our experiments consist of following the movement of an intruder as it sinks into a liquefied granular medium. The intruder is a spherical ball, $4 \mathrm{~cm}$ in diameter. We used the $123 \mathrm{D}^{\circledR}$ Design software in order to design the ball and printed it with a MakerBot ${ }^{\circledR}$ Replicator2X three-dimensional (3D) printer. The sphere is made of heated polymeric material: an acrylonitrile butadienestyrene filament (type "color true yellow"). Designing our own balls, we are able to control their effective density by adjusting the thickness of the shell layer, leaving a concentric empty sphere in the center, without adding any extra weight in the spherical shell, which allows us to keep the spherical symmetry of the intruder density. The granular medium is made of water and monodisperse spherical polystyrene beads, with a diameter of $140 \mu \mathrm{m}$ (DYNOSEEDS $^{\circledR}$ TS [51]) and density of $1050 \mathrm{~kg} \mathrm{~m}^{-3}$. The friction coefficient of this material $\mu_{\text {expt }}$ is estimated at $\mu_{\text {expt }}=$ 0.48 by measuring the angle at which a thick homogeneous layer of the material starts to slide.

The experiments shown in this paper use an intruder of density $1030 \pm 5 \mathrm{~kg} \mathrm{~m}^{-3}$. The experimental protocol is as follows. First we introduce water in a transparent PMMA cubical box of dimensions $12.8 \times 12.8 \times 12.8 \mathrm{~cm}^{3}$. We roughly fill the box up to a third of the desired final height. We next let the polystyrene beads rain down from random positions into the water, using a sieve, until the top of the beads piling up at the bottom of the container reaches the surface of the 

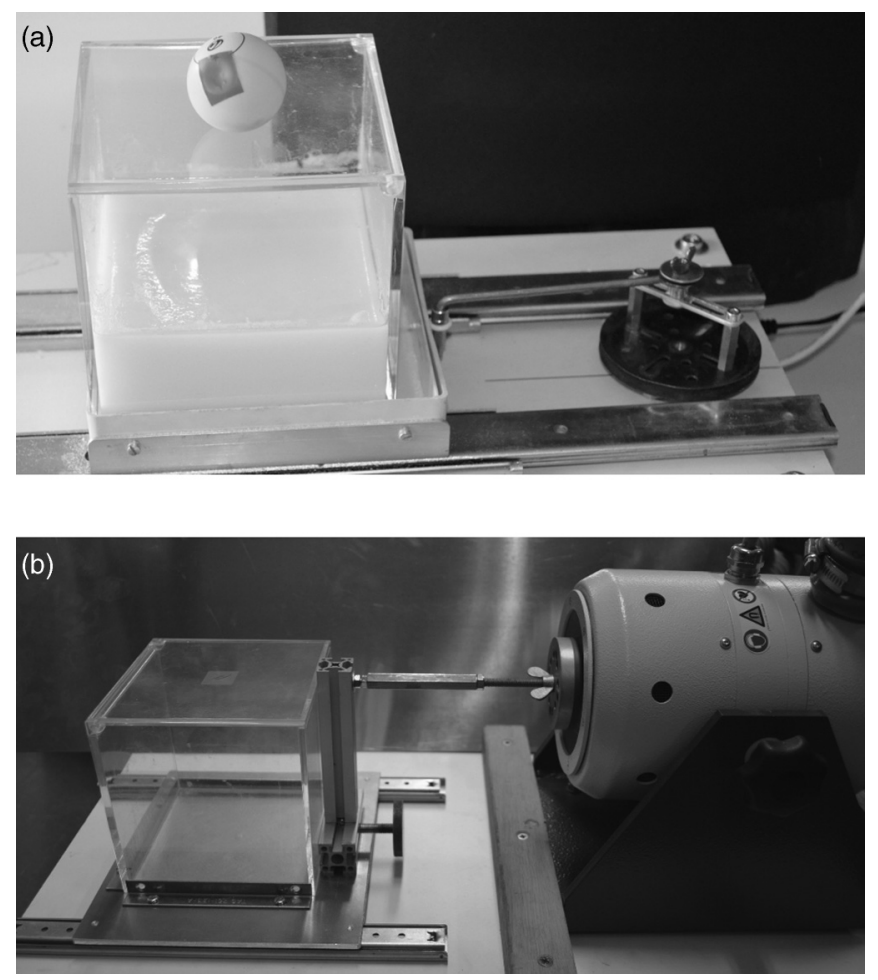

FIG. 5. Experimental setups. The mechanical part on the right exerts a horizontal movement on the box, guided by the rails. (a) Home-developed vibrator, using a Phidget ${ }^{\circledR} 1063$ PhidgetStepper Bipolar 1 and MATLAB ${ }^{\circledR}$ controls. This stepmotor provides an oscillation with an amplitude range $(\mathrm{mm})[5 ; 30]$ and a frequency range (Hz) $[0.15 ; 2.8]$. (b) TIRA $^{\circledR}$ TV51120 shaker that we used with an amplitude range $(\mathrm{mm})[0.2 ; 1.5]$ and frequency range $(\mathrm{Hz})$ $[4 ; 100]$.

water. Two versions of the setup are shown in Fig. 5, using two different vibrators reaching different powers and frequency ranges: (a) a homemade vibrator, using a Phidget ${ }^{\circledR} 1063$ PhidgetStepper Bipolar 1 and MATLAB ${ }^{\circledR}$ controls, and (b) a TIRA $^{\circledR}$ TV51120 shaker, type S51120, for higher frequencies and larger power. After $3 \mathrm{~min}$ of relaxation time, sufficient for the granular matter to settle in the wet medium, we gently depose the intruder on the surface of the medium. After another minute of relaxation, the box is horizontally shaken with a sinusoidal movement of controlled amplitude and frequency. A camera records the experiments. In the setup in Fig. 5(a) we use a Nikon ${ }^{\circledR}$ Digital Camera D5100 with an 80-mm objective recording at 25 frames/s. In the setup in Fig. 5(b) we use a fast camera Photron ${ }^{\circledR}$ SA5 with a similar objective at 20000 frames/s. The setup is illuminated by a flickerfree HMI $400 \mathrm{~W}$ Dedolight ${ }^{\circledR}$ spotlight in front of the experimental cell, next to the camera. The videos are cut into series of snapshots using the free software FFmpeg ${ }^{\circledR}$. Figure 6 presents six snapshots, corresponding to the different positions of the intruder from the beginning to the end of the shaking.

We can follow the position of the intruder inside the medium through image analysis. We use MATLAB ${ }^{\circledR}$ algorithms and based on the color of each pixel of each picture, we access the position of the pixel of the highest point of the ball. Using these data and geometrical considerations to correct for perspective

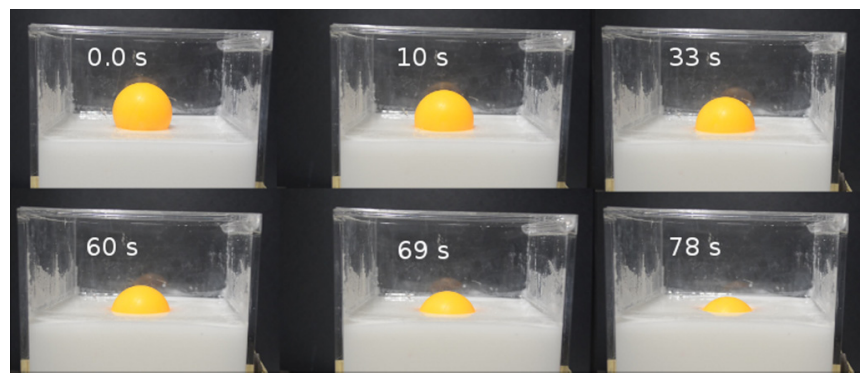

FIG. 6. Series of snapshots of an experiment, read from left to right and from top to bottom.

effects, we obtain the height of the ball above the granular medium surface.

\section{B. Numerical simulations \\ 1. Modeling principles}

Our simulations are 2D representations of the experimental setup, based on discrete-element method of molecular dynamics [57]. We use the soft-particle approach originally developed by Cundall and Strack [58] where we add a buoyancy force to account for the presence of water [45]. The simulations give access to the trajectory and transient forces acting on individual cylindrical particles immersed in a fluid inside a finite space. In order to model a 2D space of size comparable to the experiments, we need to use larger grains than the experimental ones, since the experiments performed include roughly $10^{8}$ particles, which is beyond numerical capabilities of the model described here. The behavior of each particle of mass $m$ and moment of inertia $I$ is governed by the second law of Newton and the angular momentum theorem,

$$
\begin{aligned}
\sum \boldsymbol{F}_{\mathrm{ext}} & =m \ddot{\boldsymbol{z}}(t), \\
\sum \mathcal{M}\left(\boldsymbol{F}_{\mathrm{ext}}\right) & =\boldsymbol{I} \frac{d \dot{\theta}}{d t}(t),
\end{aligned}
$$

where $\sum \boldsymbol{F}_{\text {ext }}$ and $\sum \mathcal{M}\left(\boldsymbol{F}_{\text {ext }}\right)$ are the sum of external forces and the sum of external torques acting on the particle, respectively, $\ddot{z}(t)$ is the particle acceleration, and $\dot{\theta}(t)$ is its angular velocity. Our particles are cylinders because our simulation is in two dimensions, thus, for a particle of radius $r$, the inertial momentum is $I=m r^{2} / 2$ and the mass is $m=\rho_{\mathrm{s}} \pi r^{2} l$, where $l$ is the size of the medium in the third direction. To reproduce the experimental setup, the numerical media are enclosed between walls, two vertical ones on each side and a horizontal one on the bottom (Fig. 7).

We compute the forces in the Galilean laboratory reference frame. The forces implemented on each particle are the gravity, the buoyancy force of the liquid, and the contact forces. We assume the movement of the fluid with respect to the grains to be slow enough to neglect the viscosity of the fluid. Thus, the fluid only intervenes in this model via buoyancy forces. For a particle of density $\rho_{\mathrm{s}}$, volume $V$, and immersed volume $V_{\text {im }}$, the gravity and buoyancy forces are given, respectively, by $\boldsymbol{F}_{\text {gravity }}=V \rho_{\mathrm{s}} g \boldsymbol{e}_{z}$ and $\boldsymbol{F}_{\text {buoyancy }}=-V_{\mathrm{im}} \rho_{\mathrm{w}} g \boldsymbol{e}_{z}$, where $g=$ $9.81 \mathrm{~m} \mathrm{~s}^{-2}$ and $\boldsymbol{e}_{z}$ is the downward vertical unit vector. We model the contacts between two particles with a linear 


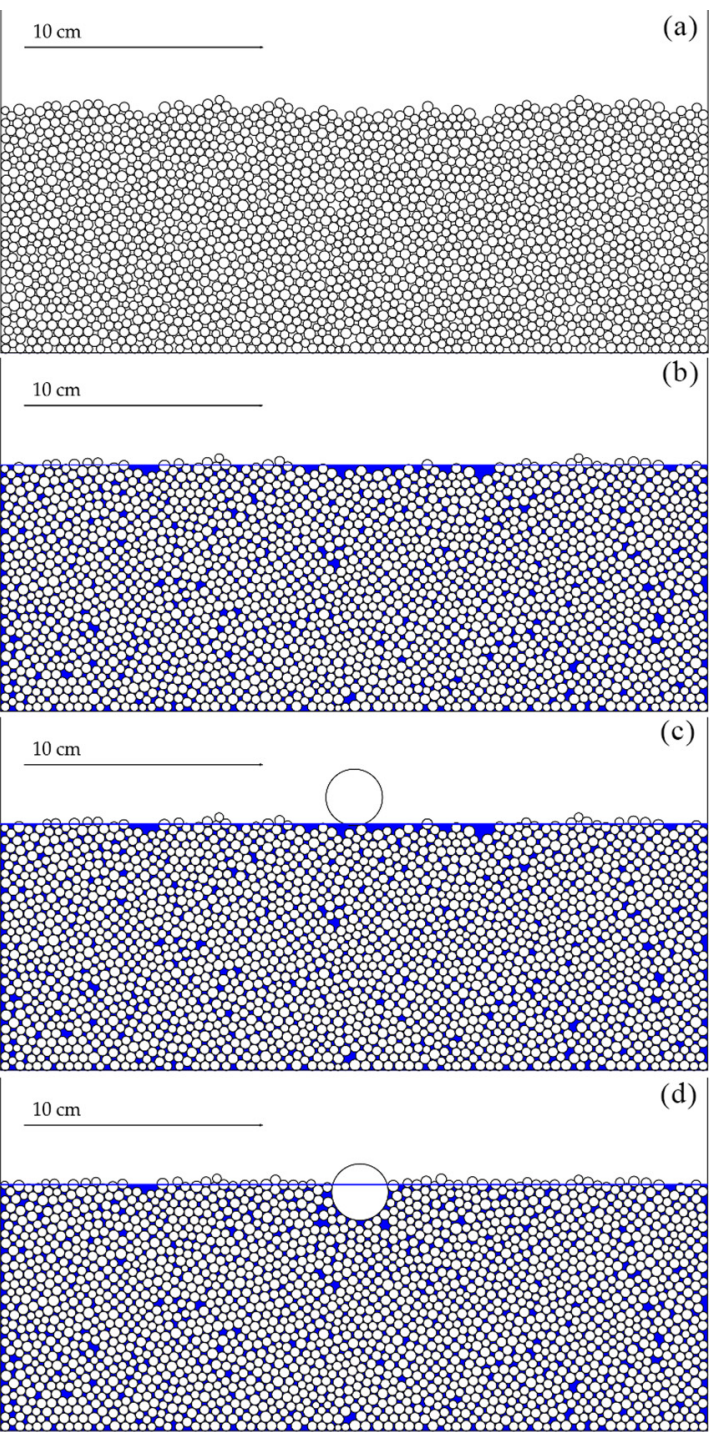

FIG. 7. The different steps to create the initial state (a) First a granular medium of 2000 particles is created. (b) Next, water is added in the porous volume (blue part). (c) Further, an intruder (of radius $12 \mathrm{~mm}$ here) is hung on the top of the medium and released. (d) Eventually the intruder relaxes to a state of mechanical equilibrium with the medium.

spring-dashpot model [58]. For each contact we take into account a viscoelastic reaction with two spring-dashpot models, one in the normal direction and one in the tangential direction in the local frame of the contact. The springs exert a linear elastic repulsion, with $k$ the elastic constant, while the dashpot models exert a dissipative force during contact as a solid viscosity, i.e., viscous damping during the shocks, with $v$ the viscosity. The particles can rotate due to friction on contacts. We implement a Coulomb friction law for each contact. If the tangential force exceeds the Coulomb criterion, we let the particle contact slide and set the tangential force equal to the normal force times the friction coefficient. The interactions between particles and the three walls are the same as between two particles, meaning that the walls have similar mechanical and contact properties as the particles. Once we have computed the sum of external forces for each particle, we deduce their acceleration and use a leapfrog form of the Verlet algorithm [57] to get the velocity and position for the next time step. The particles positions and velocity are updated and we compute the new forces.

For a realistic model, the grains need to be hard and the overlaps small. According to the differential equations governing the system, the duration of a contact is approximately given by $\sqrt{\frac{m}{k}}$, so harder grains correspond to higher $k$ and to shorter collision durations. The time step needs to be smaller than the collision duration, hence implementing harder grains means shorter time steps and longer computation times. We need a time step at least 10 times smaller than this impact duration and we are interested in having the largest time step possible to reduce computational time, which means a small enough $k$. Simultaneously the elasticity parameter $k$ has to be large enough to avoid large deformations of the particles themselves. Here we require these deformations not to exceed $1 \%$, which physically translates in the contact force (solid stress times cross-section) being lower than $k \times 0.01 r_{\text {mean }}$. The solid stress has a static and a dynamic component, the latter appearing during impact only. The static stress evolves in the medium as $\rho_{\text {eff }} g z$, with $z$ the particle depth, and the dynamic one evolves like $\rho_{\mathrm{s}} v^{2}$, with $v$ the velocity of the particles. In our system the maximum value of solid stress is attained during high-velocity collisions, when the static stress $\rho_{\text {eff }} g z$ becomes negligible compared to the dynamic stress $\rho_{\mathrm{s}} v^{2}$. The maximal velocity of the particles is attained during the preparation stage and is around $1 \mathrm{~m} / \mathrm{s}$. Eventually we need to choose a value for $k$ such that $\left(\rho_{\mathrm{eff}} g z+\rho_{\mathrm{s}} v^{2}\right) r_{\text {mean }} l<k \times 0.01 r_{\text {mean }}$, with $r_{\text {mean }}$ the mean radius of the set of particles. We choose an elasticity coefficient of $k=20000 \mathrm{~kg} \mathrm{~s}^{-2}$ and a time step of $1 \times 10^{-6} \mathrm{~s}$, which suits all our simulations. We checked that the value of the elasticity constant $k$ does not affect the behavior of the media by doubling and quadrupling its value.

\section{Our numerical granular media}

The first step is the creation of initial configurations. We define $N_{\max }$, the maximal number of particles of radius $r_{\text {mean }}$ which can fit in the width of the box $w_{\text {box }}$, as

$$
N_{\max }=\frac{w_{\text {box }}}{2 r_{\text {mean }}} \text {. }
$$

We create horizontal lines of particles by making $100 N_{\max }$ particles with random horizontal positions, at exactly $2 r_{\text {mean }}$ above the lowest altitude free of particles, and then remove overlapping ones. The particles radii follow a normal law centered around $r_{\text {mean }}$ with a standard deviation of $8 \%$ of $r_{\text {mean }}$. The line is set free to fall and reach mechanical equilibrium. This procedure goes on until the desired number of particles is reached. The final porosity is between 0.196 and 0.199 , which is characteristic of a random loose packing for a $2 \mathrm{D}$ granular medium $[59,60]$. Once this initial soil skeleton is in place, we measure the final height of the granular medium by computing the mean of the vertical position of the last layer of beads. We fix the water level at that height in order to have a saturated medium. This configuration of granular media is representative of a soil saturated with water, which is the typical soil where liquefaction and quicksand occur $[2,3,23]$. We fix the height of an intruder at the surface of this new saturated medium and release it. The size of the intruder is chosen as 6 times the linear size of the small particles so that it is significantly larger 
TABLE I. Parameters used for the simulations.

\begin{tabular}{lcc}
\hline \hline Parameter & Symbol & Value \\
\hline radius of particles & $r_{\text {mean }} \pm 8 \%$ & $2 \pm 0.16 \mathrm{~mm}$ \\
density of particles & $\rho_{\mathrm{s}}$ & $1.05 \mathrm{~g} \mathrm{~L}^{-1}$ \\
elastic constant (during shocks) & $k$ & $20000 \mathrm{~kg} \mathrm{~s}^{-2}$ \\
viscosity constant (during shocks) & $v$ & $0.3 \mathrm{~N} \mathrm{~s} \mathrm{~m}^{-1}$ \\
friction coefficient & $\mu$ & 0.6 \\
cohesion & $c$ & 0.0 \\
number of particles & $N$ & 2000 \\
time step & $d t$ & $10^{-6} \mathrm{~s}$ \\
box size & $w_{\text {box }} \times L$ & $30 \times 30 \mathrm{~cm}^{2}$ \\
radius of the intruder & $r_{B}$ & $12 \mathrm{~mm}$ \\
density of the intruder & $\rho_{B}$ & $1.0 \mathrm{~g} \mathrm{~L}^{-1}$ \\
\hline \hline
\end{tabular}

than them and remains small enough compared to the size of the box to avoid finite-size effects. The parameters used for the simulations presented in this paper are summarized in Table I. A representation of the different steps to create the final medium is given in Fig. 7, where $r_{\text {mean }}=2 \mathrm{~mm}$ and $w_{\text {box }}=30 \mathrm{~cm}$.

The next stage is the main part of the simulations. Here we impose a horizontal movement on the two lateral walls of the box. Both sides move synchronously, following a sinusoid. We record the positions and the velocities of all particles every 100 time steps.

\section{Thresholds delimiting flow types}

\section{Variables which quantify the intruder movement}

In both the computer simulations and experiments, we record the temporal evolution of the height of the intruder. From this height and the height of the granular medium we compute the immersed depth of the intruder $h(t)$ as the distance between the surface of the medium and the bottom of the intruder. The immersed volume of the intruder $V_{B \text { im }}$ is related in three dimensions to $h(t)$ by the relation

$$
V_{B \text { im }}(t)=\frac{\pi}{3}\left[3 r h^{2}(t)-h^{3}(t)\right] .
$$

To compare our results with other sizes or shapes of intruder, we will express our computation in term of $\mathcal{X}_{\text {in }}(t)$, the intruder's emerged volume $V_{B \text { em }}(t)$ normalized by its initial emerged volume $V_{B \text { em }}(0)$ and its final emerged volume $V_{B \text { em }}(\infty)$. Here $\mathcal{X}_{\text {in }}(t)$ is defined as

$$
\begin{aligned}
\mathcal{X}_{\mathrm{in}}(t) & =\frac{V_{B \mathrm{em}}(t)-V_{B \mathrm{em}}(\infty)}{V_{B \mathrm{em}}(0)-V_{B \mathrm{em}}(\infty)} \\
& =\frac{V_{B}-V_{B \mathrm{im}}(t)-V_{B}+V_{B \mathrm{im}}(\infty)}{V_{B}-V_{B \mathrm{im}}(0)-V_{B}+V_{B \mathrm{im}}(\infty)} \\
& =\frac{V_{B \mathrm{im}}(\infty)-\pi / 3\left[3 r h^{2}(t)-h^{3}(t)\right]}{V_{B \mathrm{im}}(\infty)-V_{B \mathrm{im}}(0)} .
\end{aligned}
$$

The term $V_{B \text { im }}(0)$ is the immersed volume of the intruder during the initial state, when it is at rest on the medium. Here we assume $V_{B \text { im }}(\infty)$ to be the theoretical isostatic immersed volume of the intruder $V_{B \text { im }}^{\text {equilibrium }}$, computed for an immersion in a fluid of density $\rho_{\text {eff }}$, according to Eq. (13). For all simulations and experiments $\mathcal{X}_{\text {in }}(t)$ starts at 1 and decreases
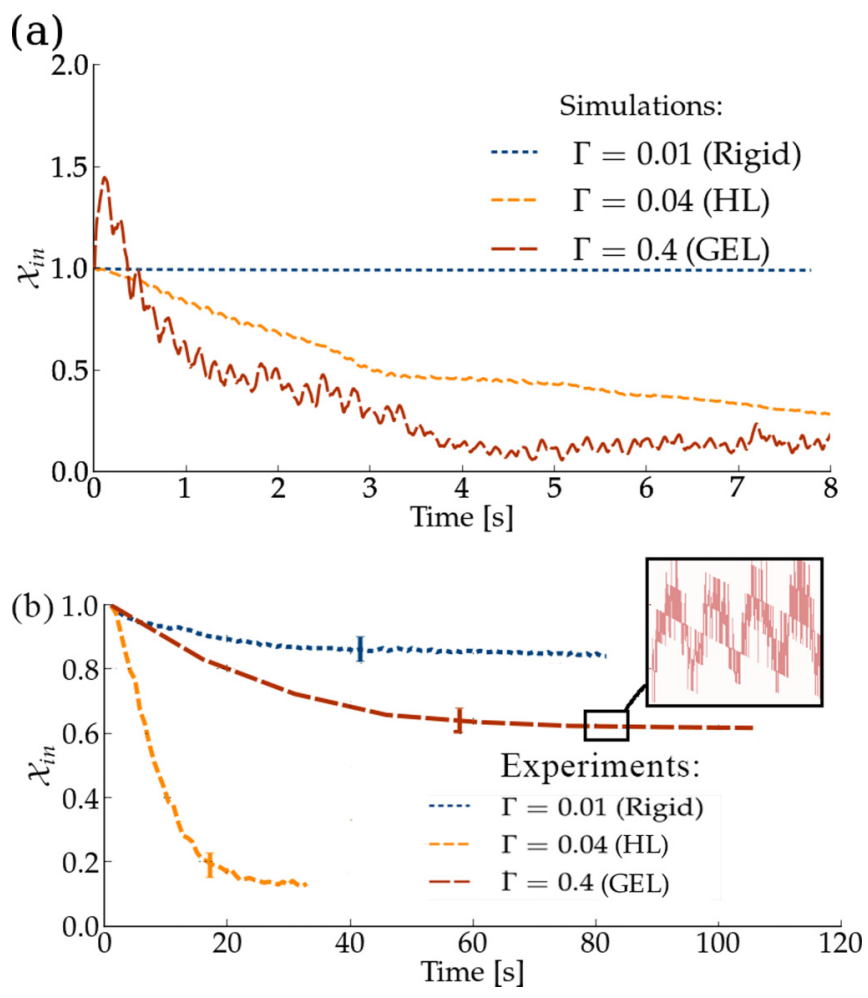

FIG. 8. Normalized emerged volume of the intruder $\mathcal{X}_{\text {in }}$ as a function of time for the three different regimes described in Sec. II B 1, in the case of (a) simulations and (b) experiments. The experiments and simulations are done in a saturated granular medium. For simulations the frequencies used are $12 \mathrm{~Hz}$ for the rigid and GEL cases and $7 \mathrm{~Hz}$ for the HL case. For experiments the frequencies used are $0.8 \mathrm{~Hz}$ for the rigid case, $1.6 \mathrm{~Hz}$ for the $\mathrm{HL}$ case, and $50 \mathrm{~Hz}$ for the GEL case.

as the intruder sinks. If the intruder reaches the isostatic equilibrium given in Eq. (13), then $\mathcal{X}_{\text {in }}(t)$ reaches 0 .

We show in Fig. 8 the evolution of $\mathcal{X}_{\text {in }}$ for three simulations and three experiments showing the typical behaviors of the three deformation regimes, rigid, HL and GEL. In the rigid cases (blue curves), $\mathcal{X}_{\text {in }}$ stays close to 1 . A small descent exists anyway, but it can be attributed to the compaction of the medium. In the HL cases (orange curves), $\mathcal{X}_{\text {in }}$ slowly decreases from 1 to a final value between 0.2 and 0 . The GEL behavior is characterized by an irregular descent of the intruder, with relatively high fluctuations around the main trend of the curve, continuously perturbing the equilibrium state. During experiments, the use of the fast camera is required to see that the intruder is continuously oscillating with high frequencies during GEL states (see the inset in Fig. 8). Even without the fast camera, one can observe with the naked eye that GEL states exhibit convection cells characterized by particles at the surface going from the middle of the box toward its sides.

In the previous well-selected cases the behavioral regimes of the system were obvious. Nevertheless, this is not always the case, especially not when the excitation is on the limit between two regimes. Hence, we need precise quantitative criteria to automatically differentiate the three regimes among all the experiments and simulations. In the following section 
we specify the exact criteria and thresholds that we use in practice.

\section{Thresholds between rigid and heterogeneous liquefaction states}

The medium is categorized to be in the rigid state when the intruder does not move significantly downward. Actually, the intruder usually sinks slightly because the medium compacts during shaking. In simulations, the medium compacts less, possibly due to the fact that the movement takes place in two dimensions and there are fewer degrees of freedom for rearrangements in two dimensions than in three dimensions. According to the observations, we categorize as rigid the experiments where $\mathcal{X}_{\text {in }}$ decreases in total less than $10 \%$ from its initial value and in the simulations where it decreases less than $5 \%$ from its initial value. The exact choice of these threshold values does not affect significantly the phase diagram we will obtain.

\section{Thresholds between heterogeneous liquefaction and global excitation liquefaction states}

When $\mathcal{X}_{\text {in }}$ decreases by more than $10 \%$ during experiments or more than 5\% during simulations, we categorize the medium state either as the HL case or as the GEL case. The distinction between these two cases is done as follows. From a macromechanical point of view, the GEL state starts when the intruder keeps oscillating around a final position without reaching a final equilibrium. Depending on the frequency, these oscillations can be small and fast or large and slow. A good criterion to determine the category is to base the distinction on the measure of the acceleration of these oscillations. This method allows us to catch the GEL cases at both low and high frequencies. When the standard deviation of the acceleration of the intruder is greater than $0.6 \mathrm{~m} \mathrm{~s}^{-2}$, the simulations and experiments are classified as GEL cases.

\section{RESULTS}

\section{A. Influence of water on soil liquefaction}

The first interesting result is the strong effect of the presence of water on the behavior displayed by the medium. To highlight the role of water in soil liquefaction, we compare the behavior of saturated and dry granular media. We first focus on laboratory experiments. We compare $\mathcal{X}_{\text {in }}$ for experimental media fully saturated to the top of the grains [Fig. 9(b)] and dry experimental media [Fig. 9(a)], shaken by the same force. For the saturated medium the transition between the rigid behavior and heterogenous liquefaction is obvious: The intruder remains on the surface for the lowest acceleration $(\Gamma=0.01$, rigid $)$, but for accelerations larger than $\Gamma \geqslant 0.04$, the intruder sinks quickly into the medium (liquefaction). On the contrary, in the dry case, for any acceleration between $\Gamma=0.01$ and $\Gamma=0.07$ the intruder does not sink. We did not observe the GEL behavior in either the dry or saturated case, since the results shown in Fig. 9 were obtained using the setup of Fig. 5(a) and the accelerations reached with this setup were too low. Global excitation liquefaction was observed for different cases where $\Gamma>1$ in both dry and saturated media, using the setup of Fig. 5(b).
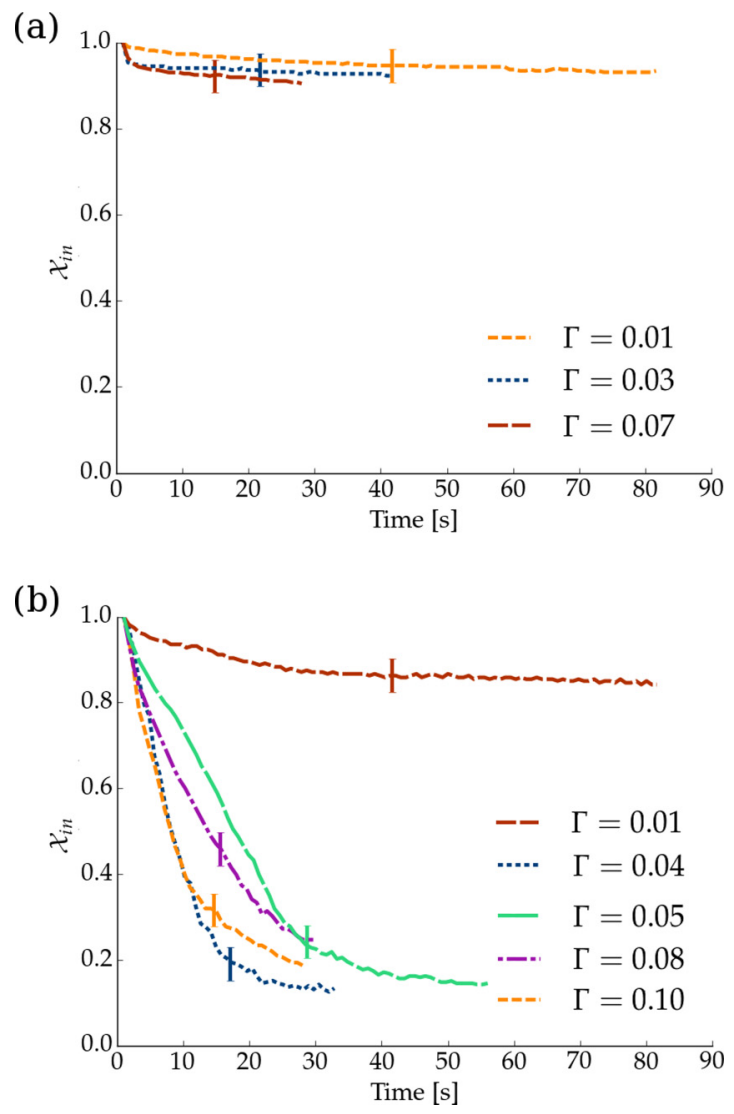

FIG. 9. Normalized emerged volume $\chi_{\text {in }}(t)$ as a function of time during several experiments (a) in a dry medium and (b) in a saturated medium. For the dry cases the horizontal shaking has an amplitude of $7 \mathrm{~mm}$ and its frequency varies from 1.5 to $3.5 \mathrm{~Hz}$. For the saturated the shaking has an amplitude of $3.5 \mathrm{~mm}$ and its frequency varies from 0.8 to $2.5 \mathrm{~Hz}$.

The same feature is observed in simulations, as shown in Fig. 10. The medium remains rigid for $\Gamma=0.01$ in both cases of dry and saturated media. An important sinking due to HL is observed for $\Gamma \in[0.05 ; 0.1]$ in the case of saturated media only. Eventually the GEL behavior can be observed on Fig. 10 for $\Gamma \in[0.3 ; 1]$. While for the saturated medium, Fig. 10(b) shows the transition between the three described regimes, rigid, HL, and GEL, the case of a dry granular medium [Fig. 10(a)] shows a direct transition from the rigid case $(\Gamma \in[0.01 ; 0.1])$ to GEL ( $\Gamma \geqq 0.3$ ), without passing through the HL regime. There is no value of $\Gamma$ where the intruder descends further beyond 5\% (for $\chi_{\text {in }}$ ) and where the standard deviation of its acceleration remains lower than $0.6 \mathrm{~m} \mathrm{~s}^{-2}$ at the same time.

These initial results confirm that the presence of water strongly promotes liquefaction and is required to produce liquefaction at moderate shaking accelerations [15]. They are qualitatively consistent with the predictions of the simple model summed up in Eqs. (6)-(8) for saturated cases and in Eq. (11) for dry cases. Both in the experiments and in the simulations, the shaken granular medium liquefies easily when water is added to the granular medium. In our simulations the presence of water is represented by local buoyancy without any compressibility or viscosity effects. Note that the pore pressure is thus increased in the saturated case with respect to the dry 
(a)

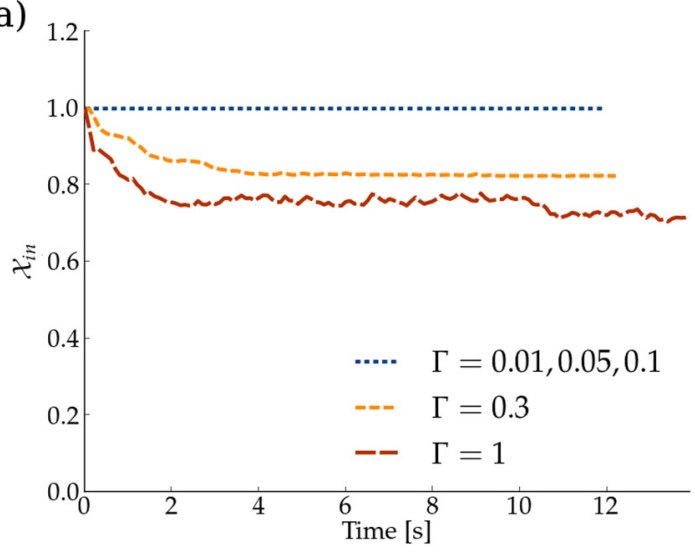

(b)

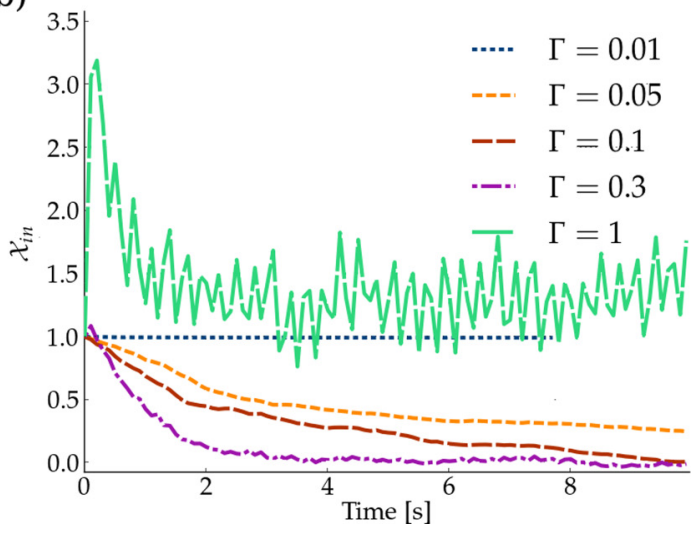

FIG. 10. Normalized emerged volume $\chi_{\text {in }}(t)$ as a function of time during several simulations run in (a) a dry medium and (b) a saturated medium. The horizontal shaking has a frequency of $12 \mathrm{~Hz}$ for the first four curves and its amplitude varies from $0.02 \mathrm{~mm}$ to $0.5 \mathrm{~mm}$. For the curve $\Gamma=1$ we used a frequency of $7 \mathrm{~Hz}$ and an amplitude of $5 \mathrm{~mm}$.

case, since it is hydrostatic, but it is not increased further during the simulations (it always stays hydrostatic): The rheology change of the saturated granular media is thus not attributable to dynamic pore-pressure rise. Liquefaction is triggered in our experiments and simulations by external shaking, with a top drained boundary condition where the water is not confined, i.e., where water can flow in and out of the surface. Inside the granular system the water acts solely through a buoyancy force and reduces the effective weight of the particles. The effective stress is reduced and consequently grains can slide more easily on each other in the presence of these buoyancy forces. The sliding motion allows liquefied deformation of the granular media. The importance of buoyancy is in enlarging the range for this sliding onset, allowing it to occur under rather low accelerations, and, more importantly, in inducing a crucial difference between the grains and the intruder: The latter being only partially immersed, the intruder-grain contacts are stronger than grain-grain contacts.

\section{B. Micromechanical point of view}

To better understand what governs the sinking of the intruder during our experiments and simulations, we focus on the deformations inside the granular medium and will here adopt a micromechanical point of view. The simulations allow
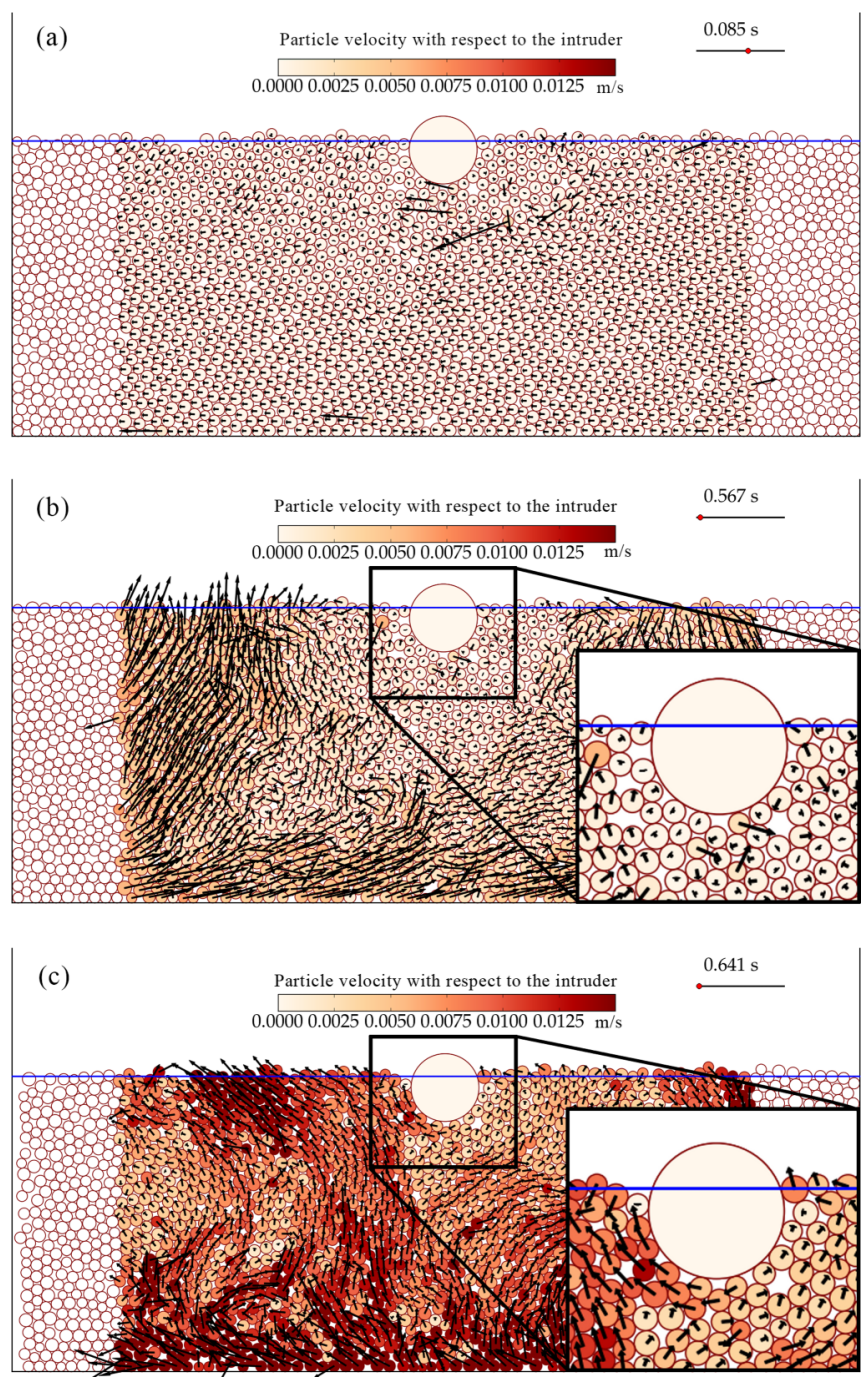

FIG. 11. Snapshots of the system configuration and velocity field for micromechanics of (a) a rigid case, (b) a HL case, and (c) a GEL case. The arrows represent the deviation velocity of the particles (velocity with respect to the intruder) and the color of the particles represent this deviation velocity norm in $\mathrm{m} \mathrm{s}^{-1}$. The blue line is the water level. The straight dark line on the top right represents the trajectory of the moving box (arbitrary scale) and the red dot the position of the box in the cycle at the time of the snapshot; the time elapsed since the start of the shaking is indicated above the line. The deviation velocity is not represented for particles close to the borders, since their behavior is dominated by the shocks against the walls and not the liquefaction process.

us to follow in detail every particle inside the medium and to investigate the physics of liquefaction. The explanation of liquefaction proposed in Sec. IIB is based on the possibility for the intruder to slide on the particles beneath it. Hence, verifying its validity necessitates considering the relative velocity between the intruder and the grains beneath. For this purpose we define the deviation velocity as the velocity of the grains in the reference frame of the intruder, i.e., the grain velocities minus the velocity of the intruder. The deviation velocity of the particles is represented in Fig. 11 for three simulations carried out in a saturated medium shaken at a frequency of 
$12 \mathrm{~Hz}$, at three different values of the normalized PGA $\Gamma$. Each snapshot shows the state of the system at a given time, with the arrows pointing in the direction of the deviation velocity and the particles' color corresponding to the deviation velocity module.

One can observe that for $\Gamma=0.01$, i.e., the rigid state shown in Fig. 11(a), the deviation velocity is almost zero in the whole medium and the intruder follows the movement of the surrounding particles. Every particle follows the imposed movement of the box and the medium does not deform. Only a few particles move or roll because of local compaction or because they are free at the surface of the medium. For $\Gamma=0.28$ the system is in the HL case, where we expect that the intruder cannot slide on the particles beneath it $(\Gamma<\mu)$, but particles can slide on each other $\left(\Gamma>\frac{\rho_{S}-\rho_{W}}{\rho_{S}} \mu\right)$. Hence, the object is not directly sliding on the surrounding particles and stays fixed to them, while the whole granular medium, far from the intruder, is able to undergo sliding and to deform easily, leading to the subsidence of the intruder and of the granular medium under it. The analysis of the deviation velocity shows that this is indeed the case: Figure 11(b) shows that the deviation velocity around the intruder is weak (under $2.5 \mathrm{~mm} \mathrm{~s}^{-1}$ ). Hence, there is no sliding between these particles and the intruder; the intruder and its neighbors move together. However, the particles further away from the intruder (a few intruder diameters) are in motion with respect to the intruder. This shows that the medium is being rearranged and, as a result, the intruder and its surrounding particles sink as a whole with respect to this far field.

Finally, for $\Gamma=0.7$ [Fig. 11(c)] the system is in the GEL regime, which can be characterized by the sliding of the intruder on the surrounding particles $(\Gamma>\mu)$. In this case the velocity deviation inside the GEL media is roughly 10 times larger than in the HL case. Under the intruder the velocity deviation of the particles is between 2.5 and $10 \mathrm{~mm} \mathrm{~s}^{-1}$ [Fig. 11(c)]. This nonzero relative velocity between the intruder and its neighbors shows that, indeed, the intruder slides on the particles beneath it, hence it behaves like any other particle of the medium. It is during this behavior that we may observe convection cells which drag the particles along cells connecting the bottom and the top of the medium. This is the case in the example of Fig. 11(c).

\section{Phase diagram controlling liquefaction occurrence and type}

Three general types of behavior have been identified and analyzed from both a macromechanical and a micromechanical point of view. We will now examine under which conditions these different behaviors occur, derive a phase diagram as a function of the control parameters, and check using experiments and simulations the theory derived in Sec. II B for the transition between these three behaviors. For this purpose, we make a systematic series of experiments and simulations at various frequencies and amplitudes.

The frequencies range from 7 to $24 \mathrm{~Hz}$ for simulations and from 0.15 to $50 \mathrm{~Hz}$ for experiments and the acceleration ranges from 0.01 to $1 \mathrm{~m} \mathrm{~s}^{-2}$ for simulations and from 0.001 to $4 \mathrm{~m} \mathrm{~s}^{-2}$ for experiments. We link each experimental and simulation run to one of the three behaviors, rigid, HL, or GEL, according
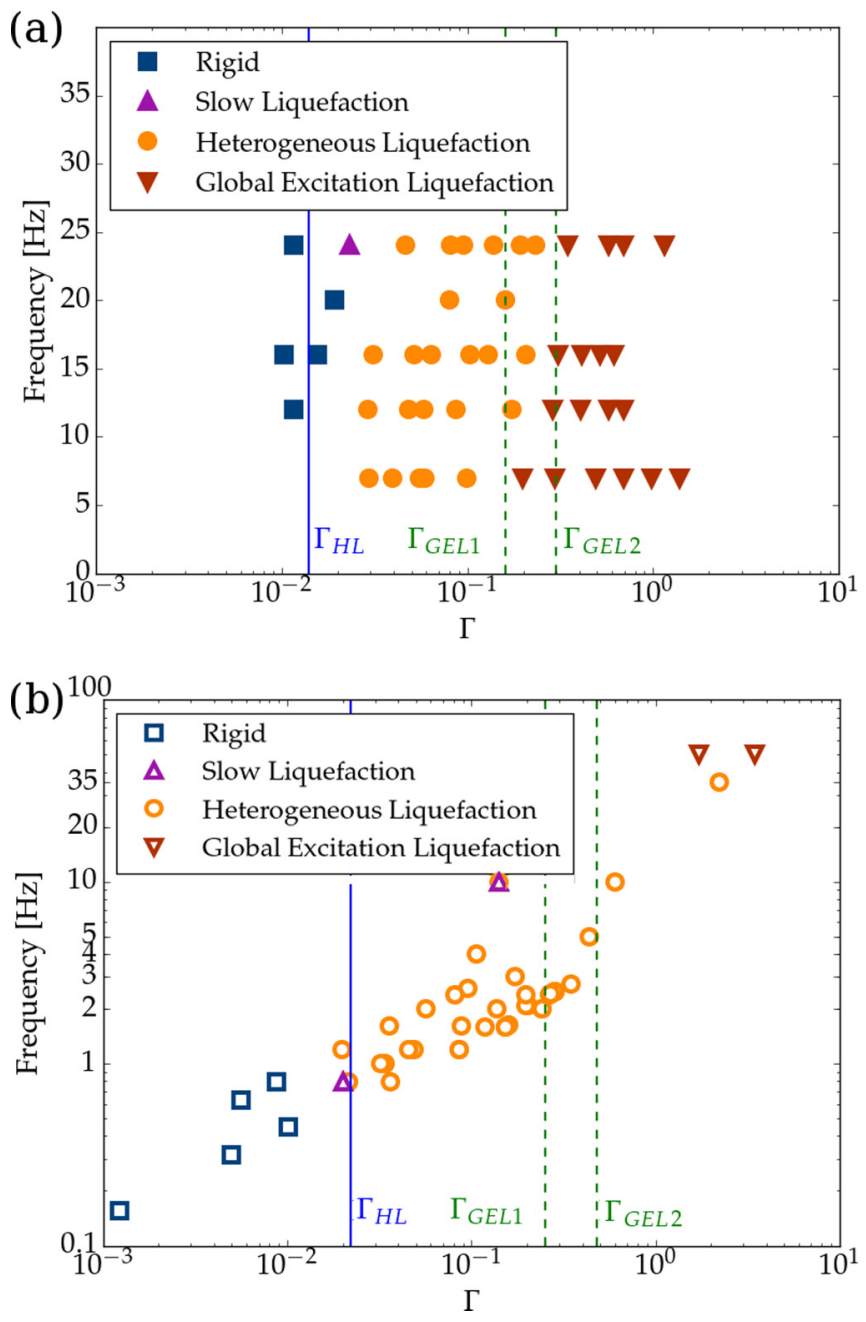

FIG. 12. Phase diagram of (a) the numerical simulations and (b) the experiments. Each simulation or experiment is plotted according to its reduced acceleration $\Gamma$ and its frequency. The behaviors are determined following the thresholds defined in Secs. III C 2 and III C 3. Squares correspond to an observed rigid behavior, circles and triangles pointing up to HL behavior, and triangles pointing down to GEL behavior. The boundaries derived theoretically in Sec. II B between these three regimes are the vertical lines: $\Gamma=\Gamma_{\mathrm{HL}}$ for the rigid-HL boundary and $\Gamma=\Gamma_{\mathrm{GEL} 1}$ and $\Gamma=\Gamma_{\mathrm{GEL} 2}$ are two possibilities for the HL-GEL boundary. These boundaries match well with the observed symbol changes, both in experiments and in simulations.

to the categorization criteria explained in Secs. III C 2 and III C 3. In the phase diagram (Fig. 12) we show the results of this systematic study for the numerical simulations [Fig. 12(a)] and the experiments [Fig. 12(b)]. There $\Gamma$ is represented on the horizontal axis and the frequency of shaking is on the vertical axis. Each simulation and experiment is plotted with a particular symbol representing the associated behavior: blue squares for rigid states, orange disks for HL, states and red triangles pointing down for GEL states, according to the thresholds defined in Sec. IIIC. A particular liquefaction behavior, represented by purple triangles pointing up and called slow liquefaction, will be further discussed in the next section. It is related to a few experiments and simulations which do not follow the same master curve as all other experiments 
and simulations. As predicted by the theory and confirmed by Fig. 12 for both simulations and experiments, the main control parameter determining whether liquefaction happens, as well as what type of liquefaction, is the value of the normalized peak ground acceleration $\Gamma$.

Two possibilities are shown for $\Gamma_{\mathrm{GEL}}: \Gamma_{\mathrm{GEL} 2}$ corresponding to the theoretical sliding threshold of an initially emerged intruder [Eq. (9) of Sec. II B] and $\Gamma_{\mathrm{GEL} 1}$ corresponding to the sliding threshold of an intruder which is initially partially immersed. Here $\Gamma_{\mathrm{GEL} 1}$ is calculated as $\Gamma_{\mathrm{GEL} 1}=\mu\left(1-\frac{V_{B \text { im }}(0) \rho_{\mathrm{W}}}{V_{B} \rho_{B}}\right)$, with the initial immersed volume $V_{B \text { im }}(0)$ of $50 \%$ for the simulations and $12 \%$ for experiments. In addition, $\Gamma_{\mathrm{GEL} 1}$ is smaller than $\Gamma_{\mathrm{GEL} 2}$ because the buoyancy applied on the intruder immersed volume reduces the acceleration needed to make it slide on the particles underneath.

Let us examine, from Fig. 12, the deviation between the phase boundaries derived experimentally or numerically and those obtained with the simple analytical model, considering $\Gamma_{\mathrm{GEL} 1}$ the threshold corrected for the initial partial immersion of the intruder. The boundary between rigid and HL states is very well reproduced by both the simulations and the experiments. Concerning the boundary between HL and GEL, the phase diagram of the simulations shows again a very good fit between theory and experiments. For the experiments, the setup limitations do not allow too many experiments at very large accelerations. A dispersion of the behavior results is observed, with a gradual transition from HL to GEL. The transition nonetheless happens at a central value around the one predicted by theory. At first order, the two theoretical boundaries $\Gamma_{\mathrm{HL}}$ and $\Gamma_{\mathrm{GEL}}$ capture very well the location of the different behaviors observed with numerical simulations and experiments. The similarity found in the results between simulations, experiments, and theory is thus satisfactory and validates the explanation proposed for the physics of liquefaction.

\section{Comparison of the final position and the isostatic position}

In the preceding section we showed that the behavior of the intruder above the shaken granular media can be sorted into three cases according to the imposed acceleration, as we expected given the theoretical analysis. We will now study what the final position of the intruder is during $\mathrm{HL}$ and the velocity at which the intruder approaches it. During the HL regime, as a first approximation, if the vertical friction forces on the intruder average to zero after penetration, the intruder will approximately approach the theoretical isostatic position dictated by its weight and the buoyancy (Sec. II B 3). The experimental and numerical setup enables us to test whether this approximation holds or fails, by measuring precisely the final vertical position of the intruder for a comparison with the isostatic position. For each simulation the ratio of the final position of the intruder to its isostatic position $h(\infty) / h_{\text {iso }}$ is represented in Fig. 13. Different symbols correspond to different behaviors observed for the simulations: rigid, slow liquefaction, HL, or GEL.

We see that when $\Gamma$ rises, the ratio rises toward 1, i.e., the intruder approaches its isostatic depth. The ratio is 0.2 for the slow liquefaction case and between 0.5 and 1 for most

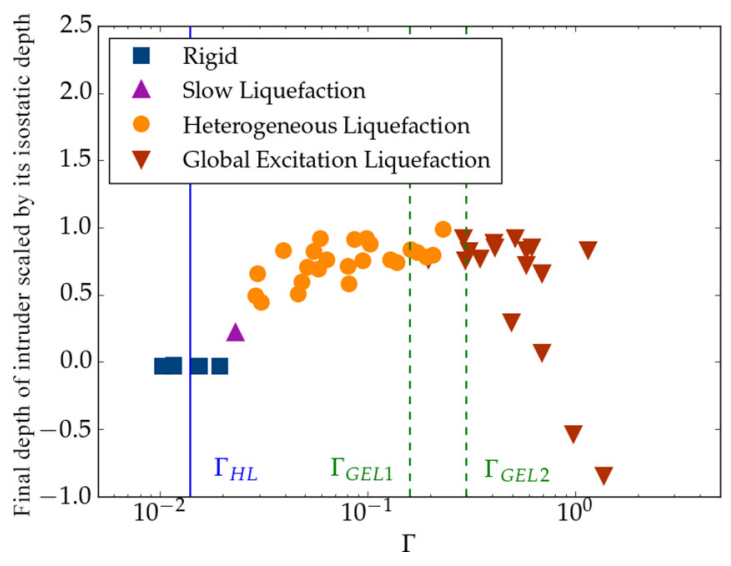

FIG. 13. Final depth of the intruder scaled by its isostatic depth $h(\infty) / h_{\text {iso }}$ as a function of the dimensionless imposed peak ground acceleration $\Gamma$. The markers correspond to the behavior of the medium, rigid, slow liquefaction, HL, or GEL.

HL cases. When $\Gamma$ exceeds $0.2-0.3$ the media displays GEL behavior and this ratio lies between 0.7 and 1 for most cases, but decreases below 0 for some cases of intense shaking, which means that instead of sinking, the intruder rises above the grains. These particular results are a demonstration of the Brazil nuts effect, where the intense shaking and the friction between grains result in a vertical force opposed to the weight of the intruder. This graph demonstrates that the isostatic position is a relatively good approximation for the final position during HL, although the results are somewhat dispersed, especially at relatively low acceleration.

\section{E. Penetration dynamics in liquefied cases}

\section{Data collapse and master curve}

To understand further the phenomenon of sinking of an object inside a liquefied granular medium, we investigate the dynamics of the intruder and how it penetrates toward its equilibrium position in the liquefactions cases. For simulations made at different amplitudes and frequencies but at the same peak ground velocity (PGV), one can observe that all the curves align together (see Fig. 14). This observation guides us to collapse the immersed volume vs time curves and establish a master curve followed by all the simulations. Considering simulations made with any amplitude and frequency, whose sinking vs time curves are shown in Fig. 15(a), we are able to collapse all curves of evolution of the sinking depth by plotting it as a function of a reduced time corresponding to the cumulated strain imposed by the oscillations, i.e., the time multiplied by the PGV [see Fig. 15(b)]. This shows that the speed of penetration of the intruder mainly depends on the peak velocity of the shaking.

\section{Exponential relaxation and characteristic time}

Concerning the shape of the sinking curves, a naturally expected shape is an exponentially or a logarithmically decreasing function. Indeed, on one hand, linear systems relax toward equilibrium following an exponential evolution. On the other hand, in nonlinear systems close to jamming or pinning, slow relaxation or creep dynamics often leads over 


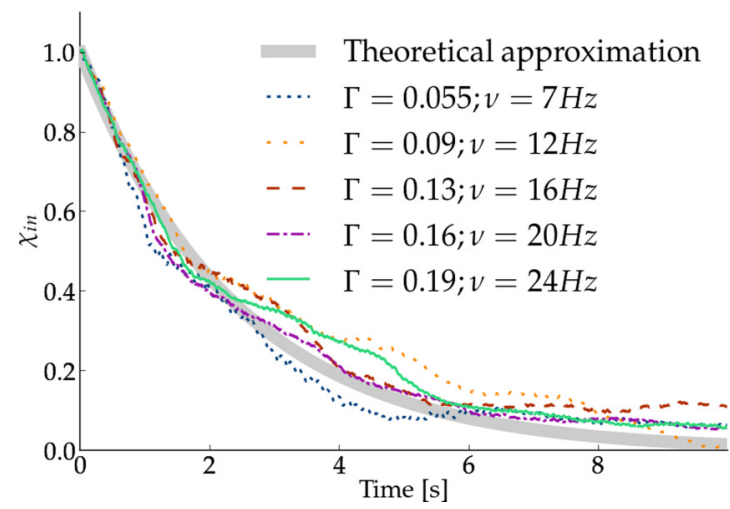

FIG. 14. Normalized emerged volume $\chi_{\text {in }}(t)$ as a function of time in simulations at different amplitudes and frequencies, but with an approximatively constant PGV of $0.012 \mathrm{~m} / \mathrm{s}$. It can be seen that simulations run with the same PGV follow the same master curve. The theoretical relaxation of the intruder for a PGV of $0.012 \mathrm{~m} / \mathrm{s}$, calculated in Sec. IV F, is plotted as a thick gray curve.

a long time to a deformation logarithmic in time. This is, for example, the case for dry grain packings compacting under vibrations [61,62], for creep in fracture propagation [63], or for deforming rocks [64]. We use semilogarithmic representations to verify if the sinking results reveal one of these behaviors. Figure 16 displays the sinking of our intruder during one simulation, characteristic of the majority of the simulation cases. Figure 16(a) shows an attempt at a logarithm fit to the sinking of the intruder and Fig. 16(b) shows an exponentially decreasing fit attempt for the same data. For both cases we present the result first with a semilogarithmic scale, where an exponential behavior would correspond to a
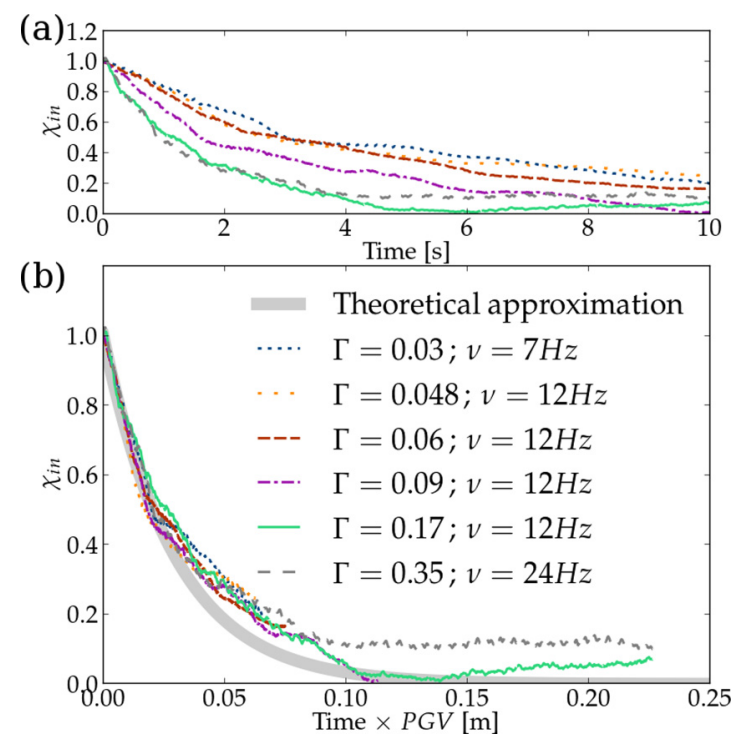

FIG. 15. (a) Normalized emerged volume of the intruder as a function of time $\chi_{\text {in }}(t)$ in a set of simulations. (b) Normalized emerged volume as a function of normalized time, multiplied by the PGV of each respective run, for the same set of simulations. The curves show a reasonable collapse on a master curve. The theoretical relaxation of the intruder is plotted as a thick gray curve. The calculation is made in Sec. IV F.
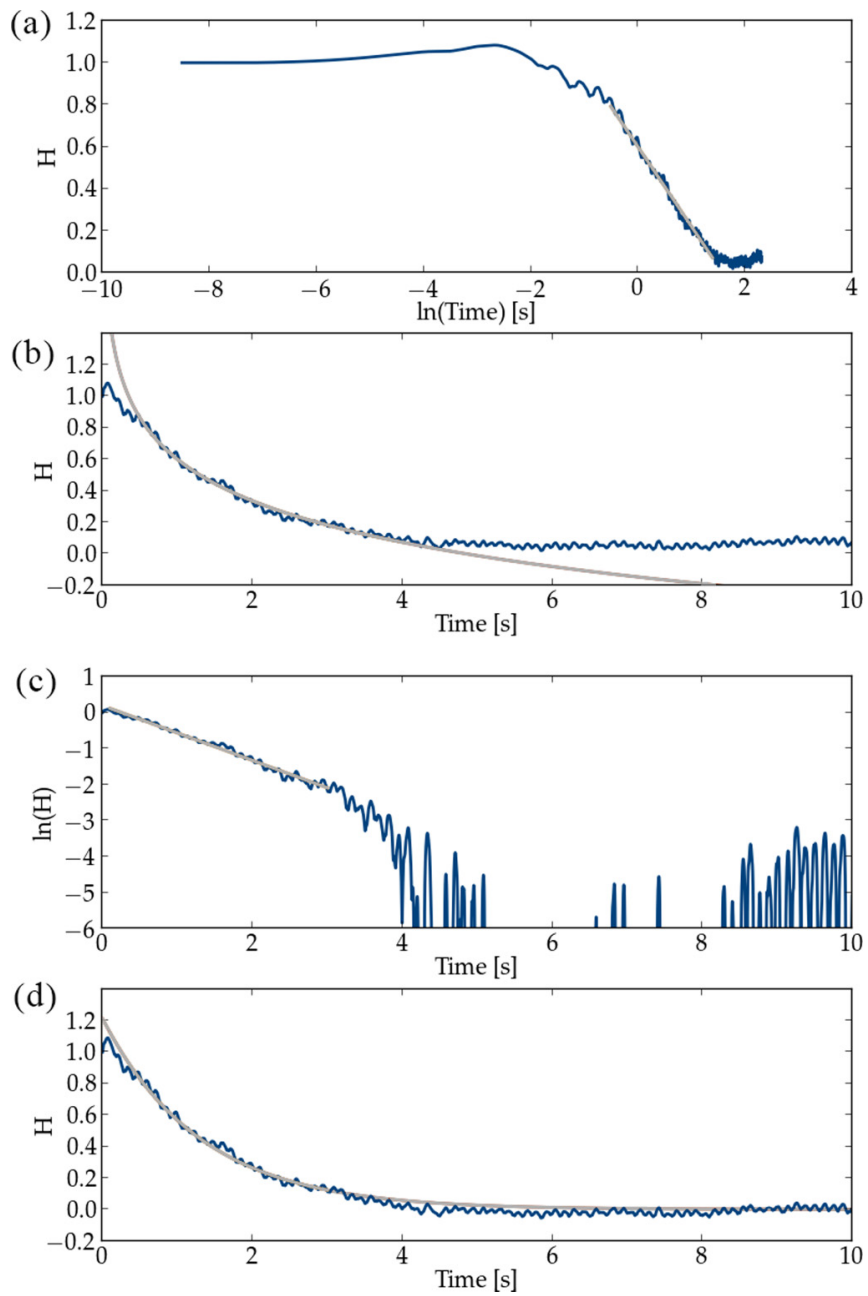

FIG. 16. Descent laws for intruders: fitting the normalized emerged height of the intruder $H(t)$ as a function of time. We apply (a) and (b) a logarithmic fit and (c) and (d) an exponential fit to the simulation data (dark blue curve). Fits are shown as light gray curves. Sinking of the intruder is best modeled by an exponential decrease of the height.

straight line, and then with a linear scale. The exponentially decreasing function fits the vast majority of the liquefied simulations, whereas the logarithmically decreasing ones fit only a few simulations whose behavior was hard to distinguish between rigid and liquefied; these correspond to the few slow liquefaction cases that will be developed in further detail in Sec. IV G. When the granular medium is well liquefied we can then assume that the intruder follows an exponential sinking toward its equilibrium position. This will be confirmed by a physical explanation later on. We apply the exponential fit to every simulation categorized as liquefied and systematically compute the half-lives. The procedure is semiautomatic. We compute the intruder normalized emerged height $H$ defined as $H(t)=\frac{h(\infty)-h(t)}{h(\infty)-h(0)}$, with $h(t)$ the immersed height previously introduced and $h(\infty)$ the immersed height at isostatic position. Here $H$ is defined by the same principle as $\mathcal{X}_{\text {in }}$ [Eq. (17)], i.e., as a normalized height which starts at 1 for every case and goes to 0 if the final immersed height of the intruder reaches $h(\infty)$, the isostatic position. We plot $H$ with a logarithmic $y$ axis, as 
(a)

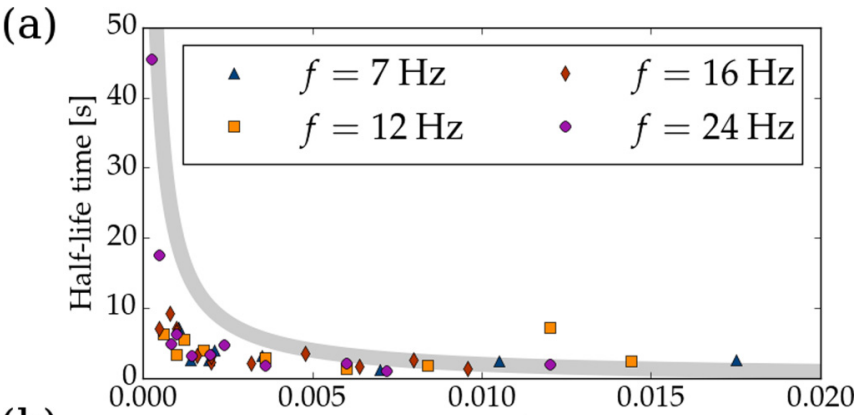

(b)

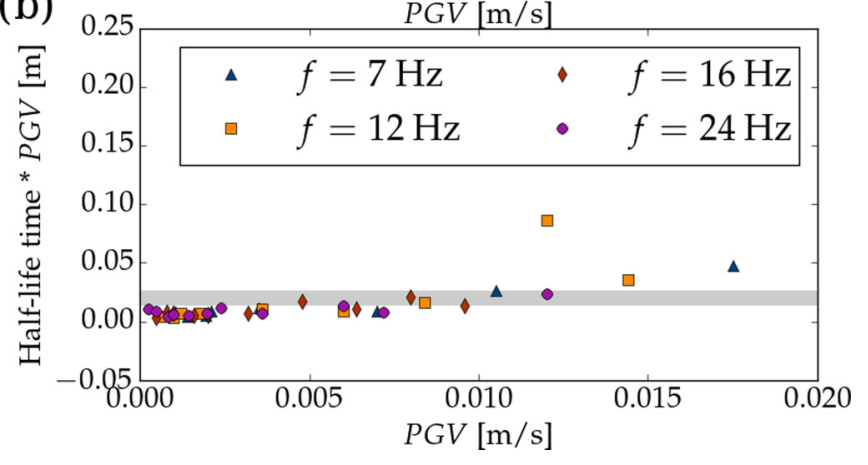

FIG. 17. (a) Half-life for sinking of intruders in simulations, as a function of the PGV. The shapes of the markers correspond to the shaking frequency. The thick gray line plots theoretical values for the half-life according to the calculation made in Sec. IVF, i.e., $t_{1 / 2}=$ $0.02 \mathrm{~m} / \mathrm{PGV}$. (b) Product of the half-life by the PGV, PGV $\times t_{1 / 2}$, as a function of the PGV. Section IV F predicts that this product is a constant.

in Fig. 16(c), and we pick manually the duration corresponding to a straight line (orange line on the plot). This duration should not be smaller than $3 \mathrm{~s}$. We then apply a linear regression to the normalized emerged height $H$, using the selected duration and a logarithmic $y$ axis, for different values of $h(\infty)$ : The linear regression is applied to 15 values of $h(\infty)$ equally spaced between the isostatic position minus $15 \%$ and the isostatic position plus $15 \%$. We keep the result which gives the largest correlation coefficient. Eventually, from the slope of the linear regression $\lambda$, we obtain the half-life as $t_{1 / 2}=\ln (2) / \lambda$.

We are now able to plot the half-life of the simulations according to the PGV of the imposed shaking (see Fig. 17). Different markers are used for different frequencies. It is clear from the collapse of Fig. 15 and from the half-life dependence of Fig. 17 that the half-life is proportional to the inverse of the PGV as all the points follow the same master curve regardless of the frequency. At first order the master curve is the inverse function. We can now present a physical explanation for the exponential sinking and for the half-life dependence on the PGV.

\section{F. Theoretical point of view}

The exponential descent of intruders into granular media has already been reported in a related study by Sawicki and Mierczyński [14]. They used real sand and a steel cylinder as an intruder. To understand the origin of this exponential behavior, we will make an approximate mechanical analysis of the granular medium rheology. There are mainly two forces acting on the intruder, apart from its weight: a buoyancy force coming from the fluid and a frictional force exerted by the solid contacts with the granular medium. Another study [27] shows that in dry granular media shaken horizontally, the frictional force opposing the intruders sinking acts locally normal to the intruder surface. Following the authors of that study, we assume that this stays valid in the saturated case, and since the penetration speed is low, we assume that the frictional force is proportional to the speed of the intruder in the medium. Newton's second law applied to the intruder and projected on the vertical axis can be phrased as

$$
\rho_{B} V \frac{d^{2} u}{d t^{2}}=-\rho_{B} V g+\rho_{\mathrm{eff}} V_{\mathrm{im}}(u) g-\alpha \frac{d u}{d t},
$$

where $u$ is the vertical displacement of the intruder, $\rho_{B}$ its density, $V$ its volume, and $\rho_{\text {eff }}$ the effective density of the saturated granular medium. We checked that $\frac{\rho_{B} V d^{2} u}{d t^{2}}$ is very small relative to the other forces. In this case, the equation of motion can be simplified as

$$
\frac{d u}{d t}=\frac{1}{\alpha}\left[-\rho_{B} V g+\rho_{\mathrm{eff}} V_{\mathrm{im}}(u) g\right] .
$$

One solution of equilibrium exists when $\rho_{B}<\rho_{\text {eff. When this }}$ solution, called $u_{\text {eq }}$, is reached, the term $\frac{d u}{d t}$ is equal to 0 . Thus

$$
\rho_{\text {eff }} V_{\text {im }}\left(u_{\text {eq }}\right)=\rho_{B} V .
$$

We focus on the dynamics near the equilibrium state, in which case

$$
V_{\mathrm{im}}(u)=V_{\mathrm{im}}\left(u_{\mathrm{eq}}\right)+\left.\frac{d V_{\mathrm{im}}}{d u}\right|_{u_{\mathrm{eq}}}\left(u-u_{\mathrm{eq}}\right) .
$$

The term $\left.\frac{d V_{\mathrm{im}}}{d u}\right|_{u_{\mathrm{eq}}}$ is linked to a characteristic surface of the intruder. It represents the disk of intersection between the intruder at the equilibrium position and the effective fluid surface. As the equilibrium position is close to total immersion, the intersection between the intruder and the effective fluid surface decreases when the intruder approaches its equilibrium and so $\left.\frac{d V_{\mathrm{im}}}{d u}\right|_{u_{\mathrm{eq}}}$ is a negative term. Thus we will write $\beta=$ $-\left.\frac{d V_{\mathrm{im}}}{d u}\right|_{u_{\mathrm{eq}}}$, where $\beta$ is positive. Equation (19) becomes

$$
\frac{d u}{d t}=\frac{1}{\alpha}\left\{-\rho_{B} V g+\rho_{\mathrm{eff}}\left[V_{\mathrm{im}}\left(u_{\mathrm{eq}}\right)-\beta\left(u-u_{\mathrm{eq}}\right)\right] g\right\} .
$$

Due to the expression of the equilibrium solution $V_{\mathrm{im}}\left(u_{\mathrm{eq}}\right)$, this becomes

$$
\frac{d u}{d t}=\frac{\beta g \rho_{\mathrm{eff}}}{\alpha}\left(u_{\mathrm{eq}}-u\right) .
$$

We finally reach a linear differential equation. Using $u_{0}=$ $u(t=0)$, the initial position, we obtain the following solution for $u$ :

$$
u(t)=\left(u_{0}-u_{\mathrm{eq}}\right) e^{\left(-\beta / g \rho_{\mathrm{eff}} \alpha\right) t}+u_{\mathrm{eq}} .
$$

So finally, after having assumed a negligible acceleration for the intruder and a frictional force proportional to the velocity of the intruder, we find that the movement of the intruder around its equilibrium position follows an exponentially decreasing law.

Concerning the half-life of penetration, our results allow us to provide an expression for $\alpha$. In Eq. (20) we have an 


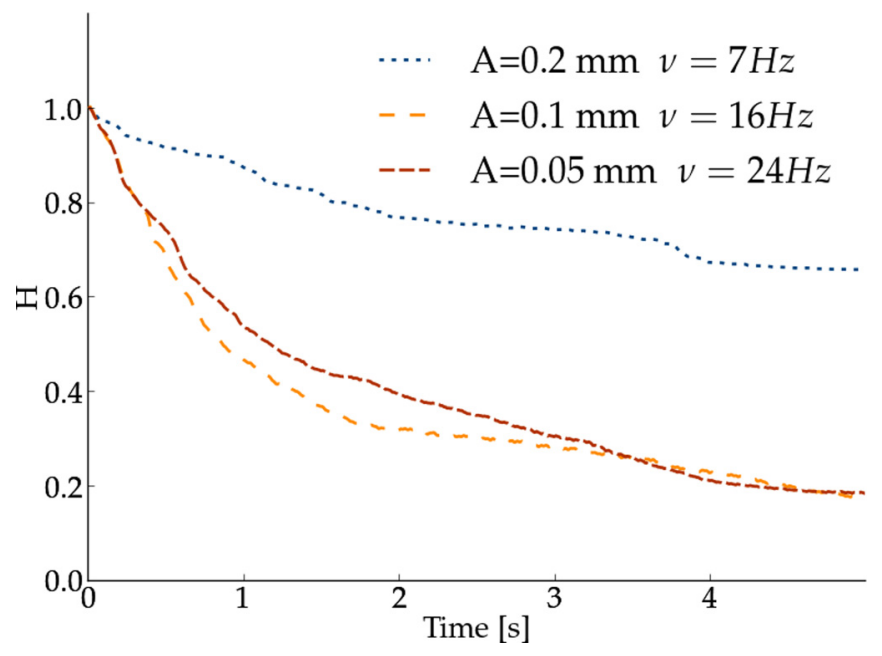

FIG. 18. Normalized height as a function of time in simulations with identical peak ground velocity. The simulation made at a frequency of $7 \mathrm{~Hz}$ does not align with the two other. It is a case of slow liquefaction.

expression for the half-life $t_{1 / 2}$ :

$$
t_{1 / 2}=\frac{\ln (2) \alpha}{\beta g \rho_{\mathrm{eff}}} .
$$

We can measure a particular half-life with Fig. 14. The simulations plotted in this figure have a PGV of $V_{0}=0.012 \mathrm{~m} / \mathrm{s}$ and the half-life is equal to $T_{0}=1.7 \mathrm{~s}$. We can assume that

$$
t_{1 / 2}=T_{0} F(A 2 \pi f)
$$

where $F$ is a function of $2 \pi A f$, the $\mathrm{PGV}$ of the simulations with $F\left(V_{0}\right)=1$. Then we use the observation made in Figs. 15 and 17, namely, that for simulations having different PGV, the alignment is obtained by multiplying the time axis by the PGV. The interpretation is that the characteristic time of the decreasing of $\mathcal{X}_{\text {in }}$, the half-life in the case of an exponential decrease, is a function of the form

$$
t_{1 / 2}=\frac{C}{A 2 \pi f},
$$

where $C$ is a constant. We can equate the two expressions (22) and (23) in order to find $C=T_{0} V_{0}=0.02 \mathrm{~m}$. In Figs. 14 and 15 we have drawn the theoretical lines corresponding to an exponential decrease from 1 to 0 with the characteristic time defined by the half-life $t_{1 / 2}$ of Eq. (23). The agreement with the half-lives extracted from the simulations demonstrates the consistency of this expression. Combining expressions (21) and (23) provides an expression for $\alpha$, which determines the prefactor of the friction law for the penetration of the intruder in the shaken granular medium, as a function of $\beta$, the cross section of the intruder close to the isostatic position, the density of the medium, and the $\mathrm{PGV} A 2 \pi f$ :

$$
\alpha=\frac{\beta g \rho_{\mathrm{eff}}}{\ln (2)} \frac{T_{0} V_{0}}{A 2 \pi f} .
$$

Here $\alpha$ may be viewed as an effective viscosity coefficient.
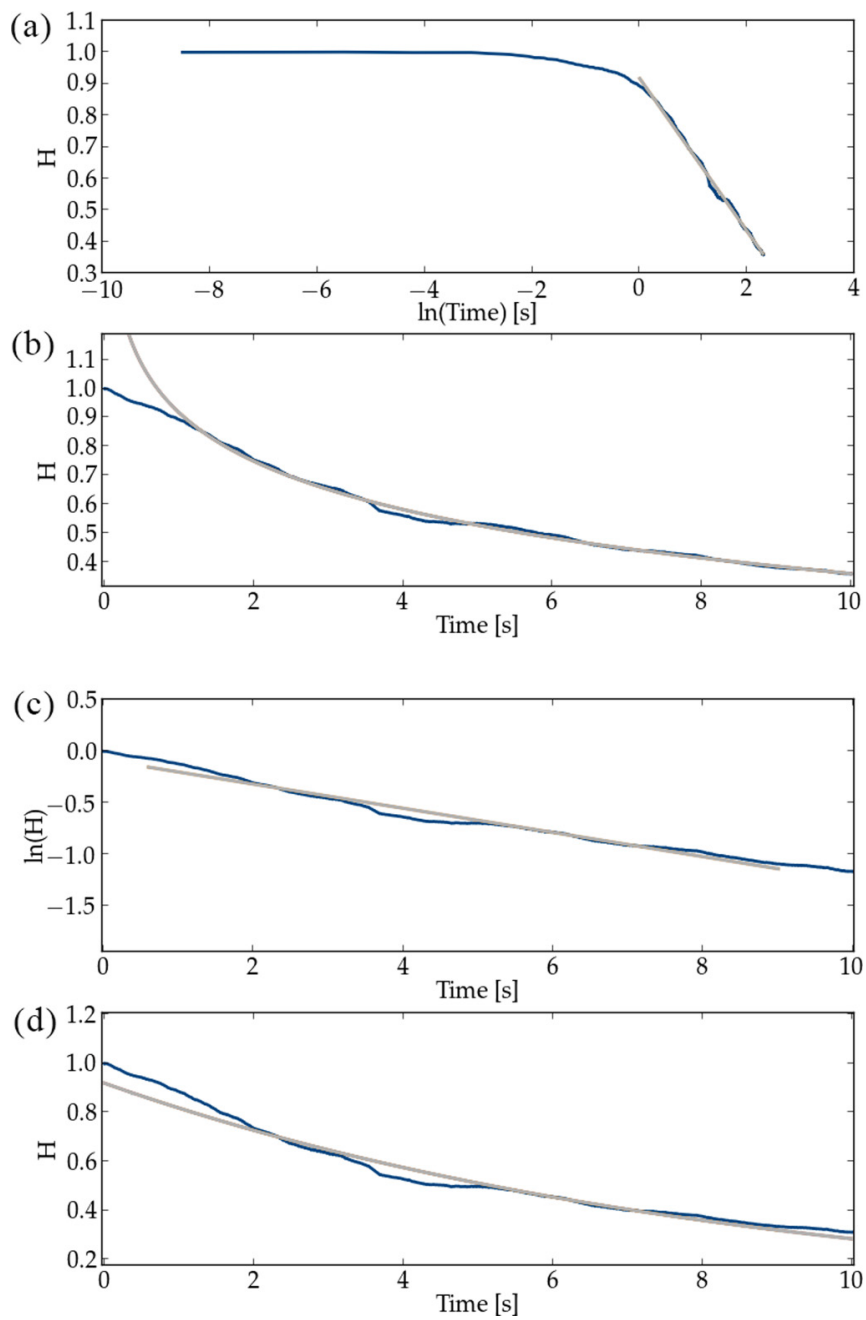

FIG. 19. Descent laws for intruders: fitting the normalized emerged height of the intruder $H(t)$ as a function of time. We apply (a) and (b) a logarithmic fit and (c) and (d) an exponential fit of the data. Fits are in light gray and data are in dark blue. We find that in some (less common) cases the emerged height follows a logarithmic decrease.

\section{G. Particular case of slow liquefaction following logarithm penetration}

The dynamics of the intruder described above predicts the behavior of most of the liquefied (HL) simulations and experiments yet fails to reproduce the behavior in a few cases. Indeed, among the simulations classified as the liquefaction state, there are a few cases where the sinking of the intruder does not follow the same trend as described above. When the intruder movement is compared to other simulations run at the same PGV, the curve does not align with the other ones, although they all have a liquefaction behavior (see Fig. 18).

We call these particular cases slow liquefaction. Observing the dynamics of penetration of the intruders in these slow liquefaction cases, we find that a logarithmic law fits better than the exponential one, as shown in Fig. 19. This is in contrast with most cases of liquefaction where the exponential decrease fits better. With the numerical simulations we find out that when the size of the numerical box is increased, these cases get less frequent. With 2000 particles, the area in the phase diagram 
where slow liquefaction appears is very narrow. This behavior agrees with recent studies on granular compaction [62], where under small excitation, granular systems get jammed and aging phenomena are observed. The stationarity of these systems is typically not reached and logarithmic relaxation is found. Our explanation for this logarithmic dynamic is that the system needs to explore rearrangement of an increasing number of particles. This can be compared to the glassy dynamic behavior and the parking lot model [65].

\section{DISCUSSION AND CONCLUSIONS}

Using a model of granular soil and a sphere representing a structure built on the top of this soil, we were able to reproduce soil liquefaction by shaking the medium. Indeed, with sufficiently strong shaking, the sphere, originally positioned on top of the medium, sinks quickly into it. Using basic physics equations taking into account buoyancy and friction between the grains, we constructed a theoretical model which predicts under which imposed shaking accelerations the sphere will sink and what its final position will be. This theoretical model predicts three different regimes for the shaken medium, depending on the friction coefficient of the material, the presence of water, and the density of the grains. When increasing the imposed acceleration, we expected subsequently a rigid regime, a heterogeneous liquefaction regime allowing the sphere to sink (representative of the conditions during seismically triggered liquefaction in nature), and a global excitation liquefaction regime where the whole medium strongly deforms. Our theoretical model to explain liquefaction has been validated by numerical simulations and experiments. Our experiments consist of 3D granular media, composed of light monodisperse beads, horizontally shaken by regular oscillations. Our simulations implement a molecular dynamics code in two dimensions with gravity, buoyancy, and contact forces. In our systems, liquefaction is controlled by the competition between the buoyancy and the gravitational forces. Because the buoyancy is not applied everywhere but only on the immersed grains, it enables the grains of the basic medium to slide on each other, while the intruder does not slide on the surrounding grains, which is at the origin of the heterogeneous liquefaction behavior we observe. We explored a broad range of accelerations and frequencies. The main conclusion is that only the peak ground acceleration of the shaking and material parameters (friction, density, and saturation) determine the behavior of the saturated granular medium. Next we showed that in the case of heterogeneous liquefaction, a spherical intruder lying on top of a granular medium sinks down to a position close to the one set by isostasy in this medium. Another conclusion relates to the dynamics of the intruder penetration. We first showed a clear data collapse in time among the sinking of the sphere with simulations, using a normalization by the inverse of the peak ground velocity. We then showed that the intruder displays usually an exponential relaxation toward equilibrium. We gave a theoretical interpretation of this relaxation and found a relationship between the effective viscosity of the fluidized medium and the shape of the immersed volume of the intruder near its isostatic position.
In a more general way, the experiments we performed show that liquefaction is possible under drained conditions. This is in agreement with recent studies [18-20]. Our simulations and experiments in effect suggest an alternative to the common view (see, e.g., $[11,12]$ ) that fluid pressure in pores between grains must rise beyond the hydrostatic value in order to produce soil liquefaction.

Our model is valid in the case of small compaction and permeable media. Indeed, for systems with large compaction and low permeability, such as clay, the dynamic pressure in pores will not be negligible anymore and viscous forces need to be added in our model as in $[66,67]$.

All the experiments and simulations that we showed were made using light particles of bulk density $1050 \mathrm{~kg} \mathrm{~m}^{-3}$ and in a noncohesive saturated medium, which increased the effect of liquefaction and allowed clear depth-of-sinking measurements. Some adaptations are needed to evaluate the liquefaction potential of a real soil. The range of accelerations for which liquefaction occurs will decrease with the use of characteristic soil particle density, and the isostatic position of an intruder in a denser medium will be more emerged than in our experimental (and numerical) media. The presence of cohesion between the particles and the fact that water often does not saturate soils up to the surface will also increase the critical acceleration needed to liquefy a soil. Moreover, the monodisperse spherical particles of our media are a chosen simplification of the setup to improve the reproducibility, but is very simplified with respect to the diversity of shapes, sizes, and densities of the particles composing a natural soil, which will certainly affect the friction parameter and can give rise to additional complexities. Our work is meant to give insight into the physical phenomenon by which soil liquefaction is triggered during an earthquake, and many perspectives are open in order to explore widely the extensions of this model, notably, by varying saturation, polydispersity, and variable densities.

As a perspective, we suggest the study of the impact of the presence of a second intruder near the intruder's penetration. Indeed, during some liquefaction events, one can notice that a building can sink or tilt while the neighboring buildings remain stable. An assumption is that the weight of one building may stabilize the surrounding soil. Another interesting line of work would be to confront our model with a broad range of grain densities and to systematically vary the level of fluid in the medium to check the fluid level impact on liquefaction. Eventually, it would be interesting to use a finite time of shaking which corresponds to the typical time of an earthquake.

\section{ACKNOWLEDGMENTS}

We appreciate helpful discussions with K. J. Måløy, E. Altshuler, A. J. Batista-Leyva, G. Sánchez-Colina, V. Vidal, G. Schäfer, Amir Sagy, Emily Brodsky, and L. Goren. We acknowledge the support of the European Union's Seventh Framework Programme for research, technological development, and demonstration under Grant Agreement No. 316889 (ITN FlowTrans), of the CNRS INSU ALEAS program, and of the LIA France-Norway D-FFRACT. We also thank Alain Steyer and Laurent Rihouey for outstanding support in building the setups. 
[1] M. E. Cates, J. P. Wittmer, J.-P. Bouchaud, and P. Claudin, Phys Rev. Lett. 81, 1841 (1998).

[2] C.-Y. Wang and M. Manga, Earthquakes and Water (Springer, Berlin, 2010), Vol. 114.

[3] N. Ambraseys and S. Sarma, Bull. Seismol. Soc. Am. 59, 651 (1969).

[4] Y. Huang and M. Yu, Nat. Hazards 65, 2375 (2013).

[5] J. Diaz-Rodriguez, S. Leroueil, and J. Aleman, J. Geotech. Eng. 118, 981 (1992).

[6] R. Waller, Effects of the March 1964 Alaska Earthquake on the Hydrology of South-Central Alaska (US Government Printing Office, Washington DC, 1966).

[7] E. Kuribayashi and F. Tatsuoka, Soils Found. 15, 81 (1975).

[8] H. B. Seed and I. M. Idriss, J. Soil Mech. Found. Div. 93, 83 (1967).

[9] M. Cubrinovski, J. Bray, M. Taylor, S. Giorgini, B. Bradley, L. Wotherspoon, and J. Zupan, Seismol. Res. Lett. 82, 893 (2011).

[10] K. Terzaghi, Theoretical Soil Mechanics (Wiley, New York, 1944).

[11] T. Youd, I. Idriss, R. Andrus, I. Arango, G. Castro, J. Christian, R. Dobry, W. Finn, L. Harder, Jr., M. Hynes et al., J. Geotech. Geoenviron. Eng. 127, 817 (2001).

[12] R. B. Seed, K. O. Cetin, R. E. S. Moss, A. M. Kammerer, J. Wu, J. M. Pestana, M. F. Riemer, R. B. Sancio, J. D. Bray, R. E. Kayen, and A. Faris, EERC Report no. 2003-06, Earthquake Engineering Research Center, University of California, Berkeley (2003).

[13] A. Sawicki and J. MierczyĹ, Appl. Mech. Rev. 59, 91 (2006).

[14] A. Sawicki and J. Mierczyński, Comput. Geotech. 36, 531 (2009).

[15] C. Clément, R. Toussaint, and E. Aharanov, arXiv:1802.04391.

[16] R. A. Green and J. K. Mitchell, in Geotechnical Engineering for Transportation Projects, edited by M. K. Yegian and E. Kavazanjian (ASCE, Reston, 2004), pp. 1961-1970.

[17] C.-Y. Wang, Seismol. Res. Lett. 78, 512 (2007).

[18] L. Goren, E. Aharonov, D. W. Sparks, and R. Toussaint, J. Geophys. Res.: Solid Earth 115, B09216 (2010).

[19] L. Goren, E. Aharonov, D. Sparks, and R. Toussaint, Pure Appl. Geophys. 168, 2289 (2011).

[20] D. Lakeland, A. Rechenmacher, and R. Ghanem, Proc. R. Soc. A 470, 20130453 (2014).

[21] S. Obermeier, Eng. Geol. 44, 1 (1996).

[22] K. Soga, Proc. ICE: Geotech. Eng. 131, 34 (1998).

[23] A. Khaldoun, E. Eiser, G. Wegdam, and D. Bonn, Nature (London) 437, 635 (2005).

[24] A. Khaldoun, G. Wegdam, E. Eiser, and D. Bonn, Europhys. News 37, 18 (2006).

[25] Y. Jiang and M. Liu, Granular Matter 11, 139 (2009).

[26] D. Lohse, R. Rauhé, R. Bergmann, and D. van der Meer, Nature (London) 432, 689 (2004).

[27] T. A. Brzinski III, P. Mayor, and D. J. Durian, Phys. Rev. Lett. 111, 168002 (2013).

[28] F. Pacheco-Vázquez, G. A. Caballero-Robledo, J. M. SolanoAltamirano, E. Altshuler, A. J. Batista-Leyva, and J. C. RuizSuárez, Phys. Rev. Lett. 106, 218001 (2011).

[29] O. Pouliquen, Phys. Fluids 11, 542 (1999).

[30] Y. Bertho, F. Giorgiutti-Dauphiné, and J.-P. Hulin, Phys. Fluids 15, 3358 (2003).
[31] S. Parez, E. Aharonov, and R. Toussaint, Phys. Rev. E 93, 042902 (2016).

[32] W. Losert, L. Bocquet, T. C. Lubensky, and J. P. Gollub, Phys. Rev. Lett. 85, 1428 (2000).

[33] N. Mujica and F. Melo, Phys. Rev. Lett. 80, 5121 (1998).

[34] D. A. Huerta, V. Sosa, M. C. Vargas, and J. C. Ruiz-Suárez, Phys. Rev. E 72, 031307 (2005).

[35] T. Pöschel and H. Herrmann, Europhys. Lett. 29, 123 (1995).

[36] A. Rosato, K. J. Strandburg, F. Prinz, and R. H. Swendsen, Phys. Rev. Lett. 58, 1038 (1987).

[37] G. Varas, J.-C. Géminard, and V. Vidal, Granular Matter 15, 801 (2013).

[38] L. S. Tsimring, R. Ramaswamy, and P. Sherman, Phys. Rev. E 60, 7126 (1999).

[39] T. Shinbrot and F. J. Muzzio, Phys. Rev. Lett. 81, 4365 (1998).

[40] M. P. Ciamarra, A. Coniglio, and M. Nicodemi, Eur. Phys. J. E 22, 227 (2007).

[41] J. L. Vinningland, Ø. Johnsen, E. G. Flekkøy, R. Toussaint, and K. J. Måløy, Phys. Rev. Lett. 99, 048001 (2007).

[42] J. L. Vinningland, Ø. Johnsen, E. G. Flekkøy, R. Toussaint, and K.J. Måløy, Phys. Rev. E 76, 051306 (2007).

[43] J. L. Vinningland, Ø. Johnsen, E. G. Flekkøy, R. Toussaint, and K. J. Måløy, Phys. Rev. E 81, 041308 (2010).

[44] J. L. Vinningland, R. Toussaint, M. J. Niebling, E. G Flekkøy, and K. J. Måløy, Eur. Phys. J. Spec. Top. 204, 27 (2012).

[45] M. J. Niebling, E. G. Flekkøy, K. J. Måløy, and R. Toussaint, Phys. Rev. E 82, 051302 (2010).

[46] M. J. Niebling, E. G. Flekkøy, K. J. Måløy, and R. Toussaint, Phys. Rev. E 82, 011301 (2010).

[47] M. Melhus and I. S. Aranson, Granular Matter 14, 151 (2012).

[48] L. Goren, R. Toussaint, E. Aharonov, D. W. Sparks, and E. Flekkøy, in Poromechanics V: Proceedings of the Fifth Biot Conference on Poromechanics, edited by C. Hellmich, B. Pichler, and D. Adam (ASCE, Reston, 2013), pp. 415-424.

[49] D. Geromichalos, M. M. Kohenen, F. Mugele, and S. Herminghaus, Phys. Rev. Lett. 90, 168702 (2003).

[50] G. Sánchez-Colina, L. Alonso-Llanes, E. Martínez, A. BatistaLeyva, C. Clément, C. Fliedner, R. Toussaint, and E. Altshuler, Rev. Sci. Instrum. 85, 126101 (2014).

[51] Microbeads AS, Skedsmokorset, Norway.

[52] A. Souriau, J. Seismol. 10, 23 (2006).

[53] S. Luding, J. Duran, E. Clément, and J. Rajchenbach, Pharm. Technol. 20, 42 (1996).

[54] C. Clement, H. Pacheco-Martinez, M. Swift, and P. King, Europhys. Lett. 91, 54001 (2010).

[55] G. Sanchez-Colina, A. Batista-Leyva, C. Clement, E. Altshuler, and R. Toussaint, arXiv:1605.08024.

[56] G. Scott and D. Kilgour, J. Phys. D 2, 863 (1969).

[57] M. Allen and D. Tildesley, Computer Simulation of Liquids (Oxford University Press, Oxford, 1989).

[58] P. Cundall and O. Strack, Geotechnique 29, 47 (1979).

[59] E. Aharonov and D. Sparks, Phys. Rev. E 60, 6890 (1999).

[60] A. Donev, S. Torquato, F. Stillinger, and R. Connelly, J. Appl. Phys. 95, 989 (2004).

[61] J. B. Knight, C. G. Fandrich, C. N. Lau, H. M. Jaeger, and S. R. Nagel, Phys. Rev. E 51, 3957 (1995).

[62] P. Richard, M. Nicodemi, R. Delannay, P. Ribiere, and D. Bideau, Nat. Mater. 4, 121 (2005). 
[63] O. Lengliné, R. Toussaint, J. Schmittbuhl, J. E. Elkhoury, J. P. Ampuero, K. T. Tallakstad, S. Santucci, and K. J. Måløy, Phys. Rev. E 84, 036104 (2011).

[64] N. Brantut, M. Heap, P. Meredith, and P. Baud, J. Struct. Geol. 52, 17 (2013).
[65] A. J. Kolan, E. R. Nowak, and A. V. Tkachenko, Phys. Rev. E 59, 3094 (1999).

[66] M. J. Niebling, R. Toussaint, E. G. Flekkøy, and K. J. Måløy, Phys. Rev. E 86, 061315 (2012).

[67] M. J. Niebling, R. Toussaint, E. G. Flekkøy, and K. J. Måløy, Rev. Cubana Fis. 29, 1E66 (2012). 